\title{
Visualizing odor representation in the brain: a review of imaging techniques for the mapping of sensory activity in the olfactory glomeruli
}

\author{
F. Pain · B. L'Heureux • H. Gurden
}

Received: 20 November 2010/Revised: 30 March 2011/ Accepted: 21 April 2011

(C) Springer Basel AG 2011

\begin{abstract}
The brain transforms clues from the external world, the sensory stimuli, into activities in neuroglial networks. These circuits are activated in specialized sensory cortices where specific functional modules are responsible for the spatiotemporal coding of the stimulus. A major challenge in the neuroscience field has been to image the spatial distribution and follow the temporal dynamics of the activation of such large populations in vivo. Functional imaging techniques developed in the last 30 years have enabled researchers to solve this critical issue, and are reviewed here. These techniques utilize sources of contrast of radioisotopic, magnetic and optical origins and exploit two major families of signals to image sensory activity: the first class uses sources linked to cellular energy metabolism and hemodynamics, while the second involves exogenous indicators of neuronal activity. The whole panel of imaging techniques has fostered the functional exploration of the olfactory bulb which is one of the most studied sensory structures. We summarize the major results obtained using these techniques that describe the spatial and temporal activity patterns in the olfactory glomeruli, the first relay of olfactory information processing in the main olfactory bulb. We conclude this review by describing promising technical developments in optical imaging and future directions in the study of olfactory spatiotemporal coding.
\end{abstract}

Keywords In vivo functional imaging - Olfactory bulb . Sensory activation - Optical imaging - Intrinsic signals . Calcium dyes

F. Pain $(\varangle) \cdot$ B. L'Heureux $\cdot$ H. Gurden Laboratoire Imagerie et Modélisation en Neurobiologie et Cancérologie, UMR8165 Université Paris Sud, CNRS, Campus d'Orsay Bat 440, 91405 Orsay, France

e-mail: pain@imnc.in2p3.fr

\author{
Abbreviations \\ BOLD Blood oxygen level-dependent \\ GCaMP Calcium-sensitive green fluorescence protein \\ CCD Charge coupled device \\ 2DG $\quad\left[{ }^{14} \mathrm{C}\right]$-2-Deoxyglucose \\ EPL External plexiform layer \\ fMRI Functional magnetic resonance imaging \\ GABA Gamma-aminobutyric acid \\ GL Glomerular layer \\ GCL Granule cell layer \\ IOS Intrinsic optical signals \\ MOB Main olfactory bulb \\ MOE Main olfactory epithelium \\ MEMRI Manganese-enhanced magnetic resonance \\ imaging \\ MCL Mitral cell layer \\ M/TCs Mitral/tufted cells \\ ONL Olfactory nerve layer \\ OMP Olfactory marker protein \\ OSNs Olfactory sensory neurons \\ OCT Optical coherent tomography \\ PGCs Periglomerular cells \\ RBCs Red blood cells \\ $\mathrm{SpH} \quad$ SynaptopHfluorin \\ TPSLM Two-photon scanning laser microscopy \\ VSDs Voltage-sensitive dyes
}

\section{Introduction}

In the brain, sensory activity is distributed among large populations of neurons that are part of functional modules responsible for the spatiotemporal coding of the stimulus. A major challenge in the neuroscience field is to image and follow the time course of activation of such large 
populations in sensory cortices with an appropriate spatiotemporal resolution. At first glance, brain tissue in vivo looks like a labyrinth of blood vessels intertwined with the neurons and glial cells that enwrap them. How can one get a significant functional signal to map population activity at large surfaces from such a complex medium? Local measurements of metabolites linked to activity using highly sensitive electrodes, such as microdialysis, and electrochemical, radiosensitive or optical probes, are insufficient to solve this technical challenge. Instead, imaging techniques developed in the last three decades that make use of optical, radioisotopic and magnetic contrast sources make it possible to solve this critical issue and are reviewed here. More precisely, we present two major families of signals that can be utilized for the imaging of sensory activity: the first class uses sources linked to cellular energy metabolism and hemodynamics such as radiolabeled glucose, and the optical and paramagnetic properties of hemoglobin; the second uses exogenous indicators of neuronal activity such as calcium and voltage-sensitive dyes, genetically encoded calcium indicators and manganese as a contrast agent for MRI. Here, we put emphasis on the physiological and physical sources of these complex signals as well as on the fascinating description of odor-evoked spatial maps and their temporal dynamics.

We present the cellular topography of the main olfactory bulb (MOB) and explain why this brain sensory structure and especially its functional units, the olfactory glomeruli, are particularly attractive for the study of sensory-evoked activation maps using functional signal imaging. Exhaustive examination of recent findings and pending questions on olfactory perception and coding in the MOB as well as the accessory olfactory system are beyond the scope of the present review and are the subject of other reviews. We suggest the reader refers to recent and excellent reviews on the structural organization of the MOB and the spatial and temporal aspects of olfactory coding [1-3]. For the sake of conciseness, we further restricted this review to imaging studies in rodents, although a very significant amount of work has been done in salamander, zebrafish and insects [4].

\section{The olfactory glomeruli: an attractive model for the study of sensory-evoked activity}

The olfactory system is a sensory system of central importance for the survival of the vast majority of living species because it allows efficient detection and identification of chemical substances in the environment (food, predators). The specificity and sensitivity of this sense has long remained a fascinating and enigmatic issue for scientists because odorants are highly complex multidimensional stimuli: the main olfactory epithelium (MOE) is sensitive to thousands of odorants varying in molecular weight, chemical groups, sorption properties, and vapor pressure. To address the problem of coding, the olfactory system uses both temporal and spatial dimensions. Electrophysiological studies conducted in the 1950s indicated that temporally dynamic patterns of MOB activity are likely to be involved in olfactory processing [5]. This finding triggered a massive series of studies that showed the cellular basis and functional importance of neuronal discharges and oscillatory patterns [3]. Evidence for spatial coding of olfactory information stemmed from early ex vivo imaging techniques from the 1980s (see section "Mapping of radiolabeled glucose uptake") which indicated that each odorant activates specific focal zones in the MOB. An important advance in the understanding of the anatomofunctional organization of the olfactory system was the first molecular deciphering of the detection and discrimination between odor chemical compounds [6]. This cardinal publication together with an impressive subsequent series of articles [7-10] outlined the basic organizational principles of the vertebrate olfactory system starting from the description of monogenic G-proteincoupled olfactory receptor expression to the combinatorial receptor coding scheme that encodes odor identities. The landmark publication in 1991 was so important and relevant for neuroscience that only 13 years after, Linda Buck and Richard Axel were awarded the Nobel Price for Medicine and Physiology (2004).

How is the MOE connected to the MOB? About 3\% of our genes are used to code for the different odorant receptors on the membrane of the olfactory sensory neurons (OSNs) in the MOE. Each OSN possesses only one type of odorant receptor and OSNs carrying the same type of receptor (about 1,000 receptors in rats) send their nerve processes to the same glomerulus (about 4,000 in rats), a well-defined microregion of discrete neuropils approximately $100 \mu \mathrm{m}^{3}$ in size (Fig. 1a). Complex interactions take place among synapses within the glomerular circuit to process olfactory information that is finally conveyed out of the MOB by the principal neurons, the mitral/tufted cells (M/TCs, Fig. 1b). Interestingly, the olfactory information does not undergo filtering through the thalamus (unlike all other sensory systems). Instead, the MOB outputs directly target the primary olfactory cortex (piriform cortex) and other limbic structures such as the amygdala, entorhinal cortex and hippocampus, thus providing the anatomical basis of the strongest memory mechanisms in the brain associating odors with context [11].

Despite this progress in the understanding of anatomical connections and organization of the olfactory glomeruli, further functional data in vivo are needed to describe how activity is distributed in space and time among odoractivated olfactory glomeruli. In this context, functional 

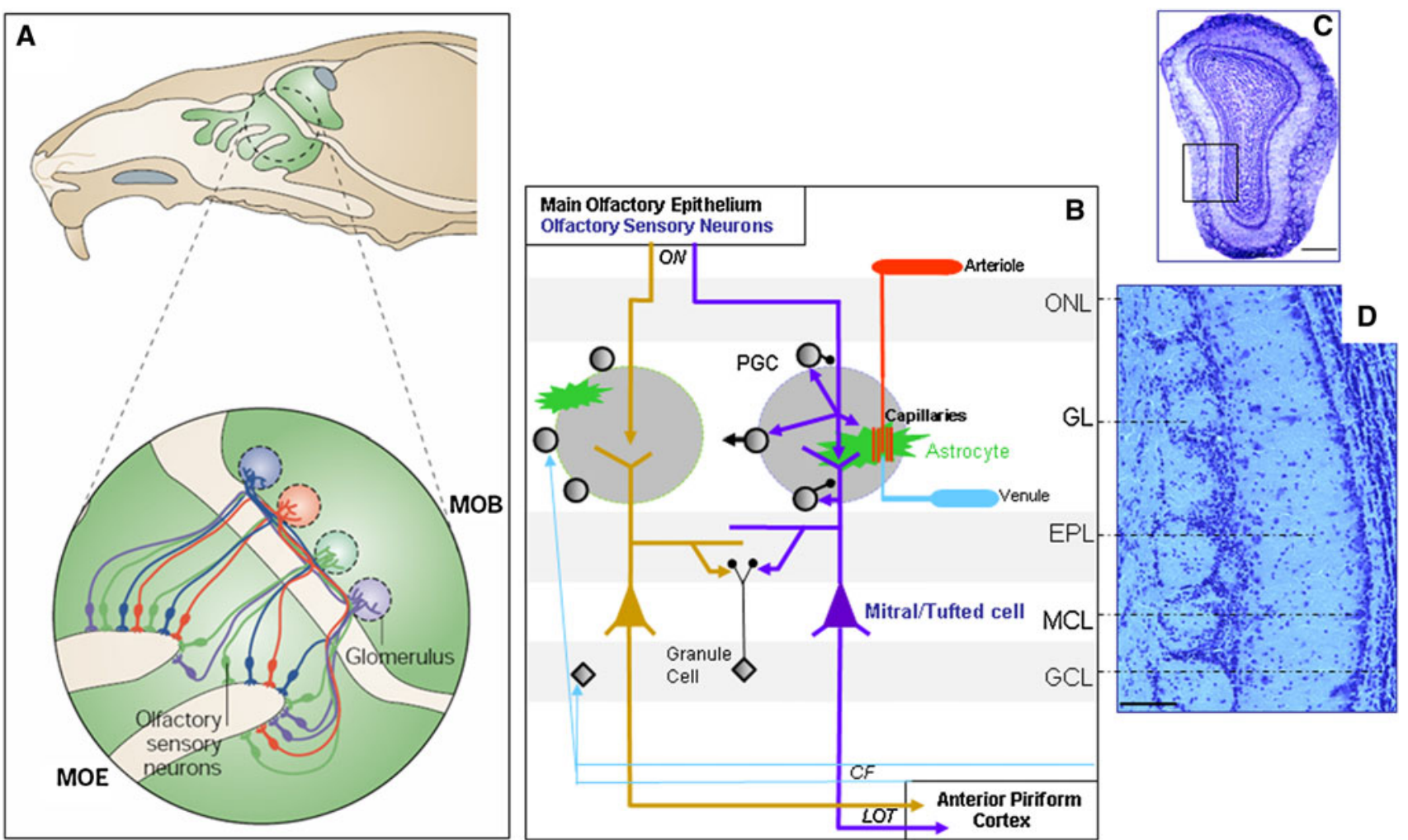

Fig. 1 The olfactory bulb, first structure for olfactory information processing in the brain. a Top: Sagittal view of a rodent head showing in green the location of the MOE at the very end of the nasal cavity and the MOB at the anterior position in the brain. Bottom: OSNs, primary receptor cells located in the MOE, which express the same odorant receptor, converge onto the same glomeruli in the MOB. b Olfactory glomeruli, the round-shaped neuropils located at the surface of the olfactory bulb, about $100 \mu \mathrm{m}$ deep just under the olfactory nerve layer $(O N L)$, are constituted by incoming convergent OSN axon fibers. In each glomerulus, projections of OSNs ramify and constitute synapses (purple arrows) with the apical dendritic tufts of principal excitatory cells (M/TCs) and inhibitory interneurons (periglomerular cells, PGCs). M/TCs and PGCs interact via dendrodendritic synapses: PGC dendrites project inhibitory GABAergic synapses onto M/TC dendrites (lower black circle), whereas M/TC dendrites make glutamatergic contacts onto PGC dendrites (purple arrow). PGCs also make GABAergic synapses onto OSN axonal terminals (upper black circle). This interneuronal population includes short axon cells which could allow lateral interactions between distant glomeruli (black arrow to the left). Numerous astrocytic projections

imaging techniques with high resolution have been successfully applied to decipher the spatiotemporal distribution of olfactory activity.

\section{Olfactory mapping using metabolic and vascular parameters as functional signals}

Rodents constantly use their olfactory system to sample their environment. This sustained activity to monitor chemical cues has an energy cost. Both experimental and are also part of this neuropil that they compartmentalize: they enwrap both synapses and blood vessels (green star-like branches) cleaning up glutamate and regulating local hemodynamics. Note that a very dense and complex vascular network is present at the glomerular level (arteriole, capillaries, venule). The dendrodendritic interactions in the external plexiform layer $(E P L)$ allowing lateral inhibition between $\mathrm{M} / \mathrm{TCs}$ are due to the activation of local inhibitory interneurons, the granule cells $(G C s)$. Each M/TC is specific to one glomerulus and sends information via the lateral olfactory tract (LOT) mainly to the anterior piriform cortex and to the limbic system without a thalamic relay. These structures send back centrifugal fibers $(C F)$ to the MOB for feedback regulation. c Coronal section of a mouse MOB (top dorsal, bottom ventral, left medial, right lateral). Inset lower magnification of image d. Scale bar $500 \mu \mathrm{m}$. d Anatomical view of MOB layers. Note the olfactory glomeruli aligned close to the MOB surface and the very thin layer of M/TCs. Scale bar $100 \mu \mathrm{m} . G L$ glomerular layer, $G C L$ granule cell layer, $M C L$ mitral cell layer, $O N$ olfactory nerve. (a Adapted from reference [1] with permission from Macmillan Publishers; copyright 2004)

simulation data show that olfactory information processing in the olfactory glomeruli has a high energy demand. Not only do glomeruli have one of the highest consumption rates of glucose in the brain [12], but they also possess the highest capillary network density [13]. The glomerular metabolic and hemodynamic system is regulated by astrocytes [14, 15] filled with glycogen [16]. Extensive modeling [17] of the energy budget in a single activated glomerulus has indicated a high energy usage in glomeruli with consumption being shared among presynaptic action potentials on OSNs and the first step of activation of 
postsynaptic receptors on M/TCs and periglomerular cell (PGC) dendrites, while subsequent dendrodendritic transmission has been found to have a lower energy consumption. Therefore, the use of metabolic and hemodynamic signals is a reliable strategy to detect neuronal activity and map the activation of glomerular modules.

\section{Mapping of radiolabeled glucose uptake}

The energy demand of brain cells is met by the production of adenosine triphosphate (ATP) in mitochondria. ATP is derived by oxidative phosphorylation of glucose which is the sole energy substrate used to support function in the adult brain [18]. Glucose crosses the blood-brain barrier via glucose transporter-1 (55-kDa-GLUT1) and enters astrocytes (45-kDa-GLUT1) and neurons (GLUT3). Glucose is present at high concentrations in the extracellular space (millimole range). The limiting step in its metabolization is phosphorylation into glucose-6-phosphate by hexokinase in the cytosol. To quantify the absolute local glucose consumption rate and how it changes following neuronal activation, ex vivo autoradiography using the ${ }^{14} \mathrm{C}$-labeled glucose analogue $\left[{ }^{14} \mathrm{C}\right]-2$-deoxyglucose (2DG) was developed. The technique was initially proposed and validated in the conscious and anesthetized rat by Sokoloff et al. in a seminal paper [19]. It relies on the metabolic accumulation of 2DG within cells after transport and phosphorylation, where the tracer does not undergo further glycolytic metabolization and is thus trapped inside the cytoplasm of all cells. When the maximum accumulation of the tracer in tissues is reached, approximately $40 \mathrm{~min}$ after injection, the animal is killed, and the target structures are revealed by autoradiography. The technique allows estimation of the local cerebral metabolic rate of glucose (LCMRGlu) given knowledge of the tracer accumulation at time $t$, the timecourse of 2DG and glucose in the arterial plasma from the time of tracer injection to time $t$, and the rate constant of glucose transport from plasma to tissue, tissue to plasma and the phosphorylation rate in the tissue of interest.

Soon after development of the technique, 2DG imaging was applied to the mapping of glucose consumption in the MOB and cortex of rabbit and rat [20]. It was used later in rat pups [21, 22] for the study of the spatial coding of odorants where authors demonstrated that unique patterns of glomeruli are activated for given odorants. The technique has been used extensively to study the influence on the odor representation of molecular features such as odorant molecular length and functional groups [12, 2325] and odorant concentration [12] in young rats (about 3 weeks old). Interestingly, using enantiomeric odorants that induced very close 2DG spatial maps, Linster et al. $[26,27]$ showed that while rats have some difficulty distinguishing — or even fail to distinguish — these stimuli in simple nonassociative learning tasks, they are fully able to discriminate them when engaged in differential reinforcement tasks.

The main drawback of the method is that it requires scarifying the animal. Thus, only a static image can be obtained for a single odor at a single concentration per experimental animal, making it impossible to access the temporal aspects of the response to odorant stimulation. Furthermore, the absolute quantitation of LCMRGlu requires several assumptions [28]: the glucose consumption is at steady state, the rate constants do not vary over time, the loss of tracer in tissue due to glucose phosphatase activity is negligible, and the ratio of 2DG to normal glucose consumption (called lump constant) is known and constant. However, values for the rate and the lump constants, which vary according to the physiological state and the brain structure, have not been evaluated in the MOB. In addition, high levels of phosphatase activity have been measured in the MOB [29] compared to neocortical tissues, suggesting important loss of tracer during accumulation. Consequently, most studies mapped activated areas in a qualitative manner even though a quantitative parameter ("z-score") was introduced [23]. Although interindividual differences in glomerular arrangements have limited the spatial resolution of the resulting odor maps [12], registration of autoradiograms with defined anatomical landmarks provides 3D views of the activated MOB for many odorants that Leon and Johnson present in their impressive open database ("Glomerular Activity Response Archive" http://gara.bio.uci.edu/index.jsp). 2DG uptake maps during sustained MOB activation and reconstructed relative glucose uptake maps are shown in Fig. 2.

Mapping of hemodynamic signals with magnetic and optical techniques

\section{Blood oxygen level-dependent functional magnetic resonance imaging}

Oxygen is delivered to brain tissues through the blood circulation and its local concentration depends on advection and diffusion movements. Parameters involved in its extraction are the local blood velocity and partial pressure in the capillaries, as well as the flux of oxygen through permeable blood vessel walls and its diffusion into cells and consumption within the mitochondria [31]. MRI has the tremendous advantage of coupling T1-weighted anatomical imaging with $\mathrm{T}^{*} 2$-weighted functional imaging, and thus 3D anatomofunctional images in the same animal can be superimposed. Although many different endogenous functional signals are under investigation for brain imaging with MRI (for a state-of-the-art review see reference [32]), Functional MRI (fMRI) is classically achieved using blood 
Fig. 2 Mapping of 2DG uptake during sustained MOB activation. Top left: Normalized $\mathrm{z}$-score flattened patterns of the whole bulb in a ventral-centered representation for three odorants in groups of 3-week-old rats $(n=10)$. Top right:

Topographic explanation of the flattened map (details of the method in reference [23]).

Bottom: Reconstructed relative glucose uptake maps. GL/SEZ glomerular layer/subependymal zone (area with no specific uptake). (Adapted from reference [30] with permission from Oxford University Press)
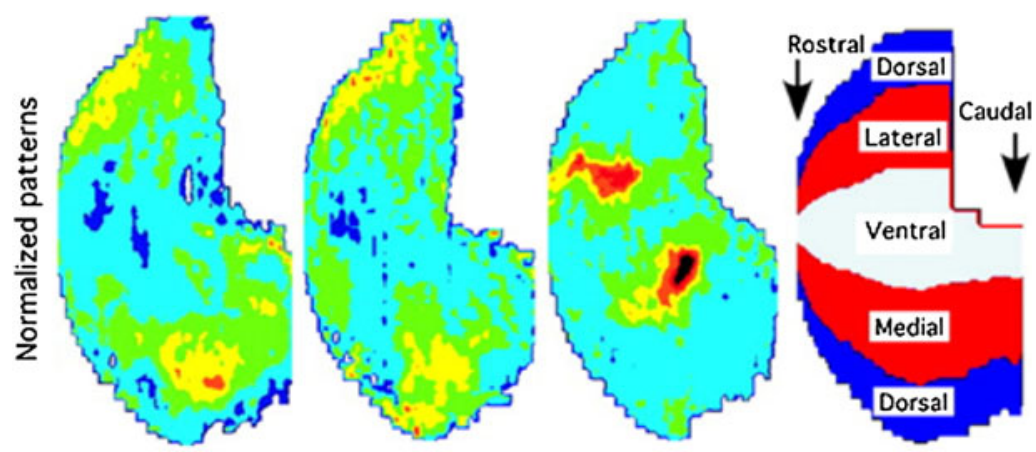

benzaldehyde

L-carvone

decanal
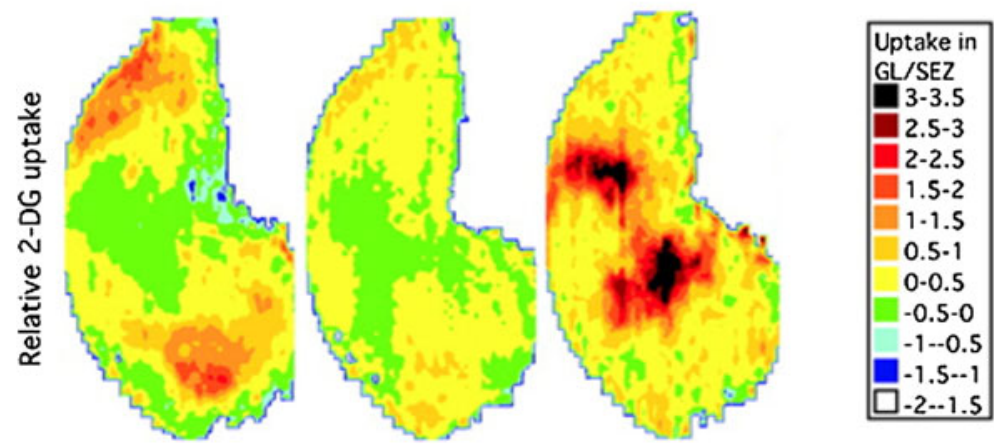

oxygen level-dependent (BOLD) contrast [33]. These signals arise from alteration in the local magnetic susceptibility due to changes in the relative concentration of oxyand deoxyhemoglobin. Changes in local blood volume and/ or oxygenation following brain activation translate into variations in the BOLD signal, which represents only an indirect measure of neuronal activity. This indirect relationship has triggered an intense experimental and modeling effort to elucidate the coupling mechanisms between cellular activity, energy demand and local hemodynamic changes [34, 35].

fMRI images of the MOB in the rodent were first obtained in the late 1990s using a 7-T magnet and a surface coil antenna adapted for small animals [36]. The obvious advantage of the fMRI technique over 2DG imaging is its ability to image the same animal repeatedly in vivo. Despite much coarser spatial and temporal resolution compared to functional optical techniques (see following sections), fMRI also has the unique advantage of being able to map not only the dorsal but the whole MOB in vivo. The majority of studies involved an odorant exposure exceeding minutes [36-39] and had a limited spatial and temporal resolution $(0.01 \mu \mathrm{l}$ and 8-30 s, respectively [37]). Acquisition at three different spatial resolutions, obtained by manipulating in-plane resolution and slice thickness while the center-of-mass of each slice remains in the same location, allows mapping at high resolution with voxel volumes down to $0.001 \mu \mathrm{l}$ [38]. In all studies, odor stimulation elicited BOLD signals in the olfactory nerve layer (ONL), glomerular layer (GL) and external plexiform layer (EPL). Qualitative examination showed activated areas symmetrically spread in the two hemispheres of the MOB. Focal areas of activation could be clearly seen with an increase in the BOLD signal up to $20 \%$ relative to the no-odor condition. The spatial patterns of activation were odorant-specific and reproducible for repeated stimulation [40]. Interindividual variations have been quantified [41] and show rough conservation of the spatial activation pattern.

Mapping functional signals with fMRI involves a tradeoff between spatial resolution and sensitivity, which ultimately defines the temporal resolution. On the one hand, mapping at the glomerular resolution has been achieved with a 2-min temporal resolution [38], and the use of a short stimulus duration combined with a fast imaging sequence has allowed researchers to follow the dynamics of MOB activation with a 1-s temporal resolution but at the expense of a spatial resolution exceeding the glomerular unit [42]. The high temporal resolution of this study allowed precise examination of the dynamics of the odor-elicited BOLD signal as shown in Fig. 3. Overall, it fits the human canonical hemodynamic response function with an onset about $2 \mathrm{~s}$ after the beginning of odor presentation, a peak 6-9 s later and return to baseline $15 \mathrm{~s}$ after stimulation. An exciting possibility is the use of the technique in the study of the plasticity of spatial patterns in the MOB during learning using a longitudinal fMRI follow-up of MOB activity during the different steps of odor task acquisition. 
Fig. 3 Mapping of MOB activation using BOLD-fMRI. Left: Coronal BOLD image through a rat MOB. Significant odor-activated zones are represented in red. Right: Timecourse of odor-induced BOLD signal changes showing delayed activation of the ventral region. (Reprinted from reference [42] with permission from Elsevier; copyright 2007))

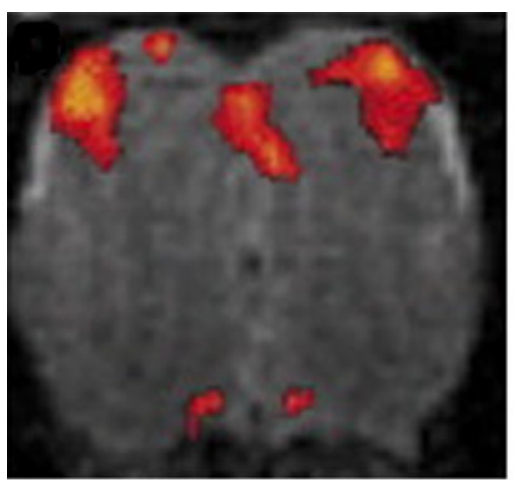

Contrast t-Map

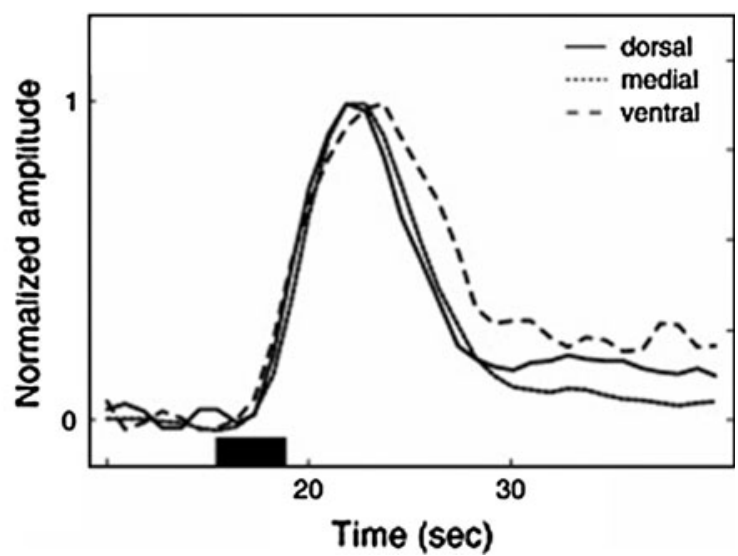

\section{Intrinsic optical signals}

The sources of contrast used in intrinsic optical signal (IOS) imaging encompass local changes in blood saturation in oxygen and blood volume which govern the local absorption of light, and rapid changes in the scattering properties of tissues following activation. The technique uses the local changes in light reflectance of tissues and was first developed and validated in mammals to map the activation of the visual cortex in the cat and the whisker barrels in the rat [43]. The use of in vivo IOS to record odor maps at the surface of the rodent MOB was demonstrated more than 10 years later in rats [44-46] and mice [47, 48]. The main advantage of the technique compared to $2 \mathrm{DG}$ autoradiography is that multiple IOS maps for different odorants can be acquired in the same animal. IOS imaging provides higher spatial resolution (below $100 \mu \mathrm{m}$ ) and temporal resolution $(500 \mathrm{~ms})$ than fMRI. Local activation responses in round areas with dimensions less than $100 \mu \mathrm{m}$ at half-maximum of peak intensity can be easily resolved. In addition, the amplitude of IOS is very high in the MOB (up to $1 \%$ of reflectance intensity changes for a mild stimulation, about ten times more than in the visual or somatosensory cortex [45]) allowing odorant representation maps to be obtained in single trial experiments. These stunning resolutions of IOS have been attributed to the well-defined anatomy of the glomeruli, the low depth of the GL [44] and the dense capillary network [47, 48]. Instrumentation required for IOS signal recording is relatively simple and affordable. As shown on Fig. 4, the set-up is composed of an optical apparatus (stereomicroscope or dual lens macroscope [49]) suited for imaging of a wide field (about $10 \mathrm{~mm}^{2}$ or more) with a working distance of several centimeters adapted for in vivo studies, where the preparation needs to be checked regularly. Light is shone onto the tissues using a stabilized light source and associated filter wheel and a scientific 12-bit cooled chargecoupled device (CCD) camera with low noise and high dynamics is used to record the faint changes in the intensity of light reflected by the tissues. A cost-effective IOS recording set-up using state-of-the-art hardware and software has been described by Harrison et al. [50].

Due to its high spatial resolution, IOS imaging allowed a major breakthrough in the olfactory field, not only because it allowed confirmation of the functional principles derived from the molecular organization of OSN projection patterns described in the 1990s (bilateral symmetry, local clustering, local variability), but also because it was the first technique to show chemotopy at a fine spatial scale for different odorant concentrations, chemical groups or mixtures which are unpredictable from molecular studies. Superimposition of anatomical map derived from loading of glomeruli with the voltage-sensitive dye RH414 in mice [47] or cytochrome oxidase staining in rats [45] has shown that activated areas match anatomically defined glomeruli in size and distribution.

Reproducible maps of changes in light reflectance relative to the no-odor condition (clean air puff stimulation) have been obtained for several odorants, and with different intensities and durations of stimulation [47]. Odor-evoked activities for the same odorants were detected in the same regions of the dorsal MOB with similar findings in different animals, but the number, position and intensity of activated glomeruli within these areas showed significant variations [46, 47, 51]. Approximate mirror activation patterns are observed between the two bulbs [47]. The threshold of activation varies among glomeruli, but all activated glomeruli show a sigmoid shaped dose-response curve when the amplitude of the signals are plotted against the $\log$ of the concentrations ranging from $0.001 \%$ up to $100 \%$ [52]. On the basis of these results, chemotopy rules for odor-elicited maps were proposed: subtle changes in odorant structure such as carbon chain length, configuration of carbon chains and functional groups led to distinct activity patterns $[44,46,53]$. The technique was used to study the coding and perception of enantiomers [54], odor mixture [55] and natural and territorial odorants [56, 57]. The limited penetration of light into brain tissue is the main 

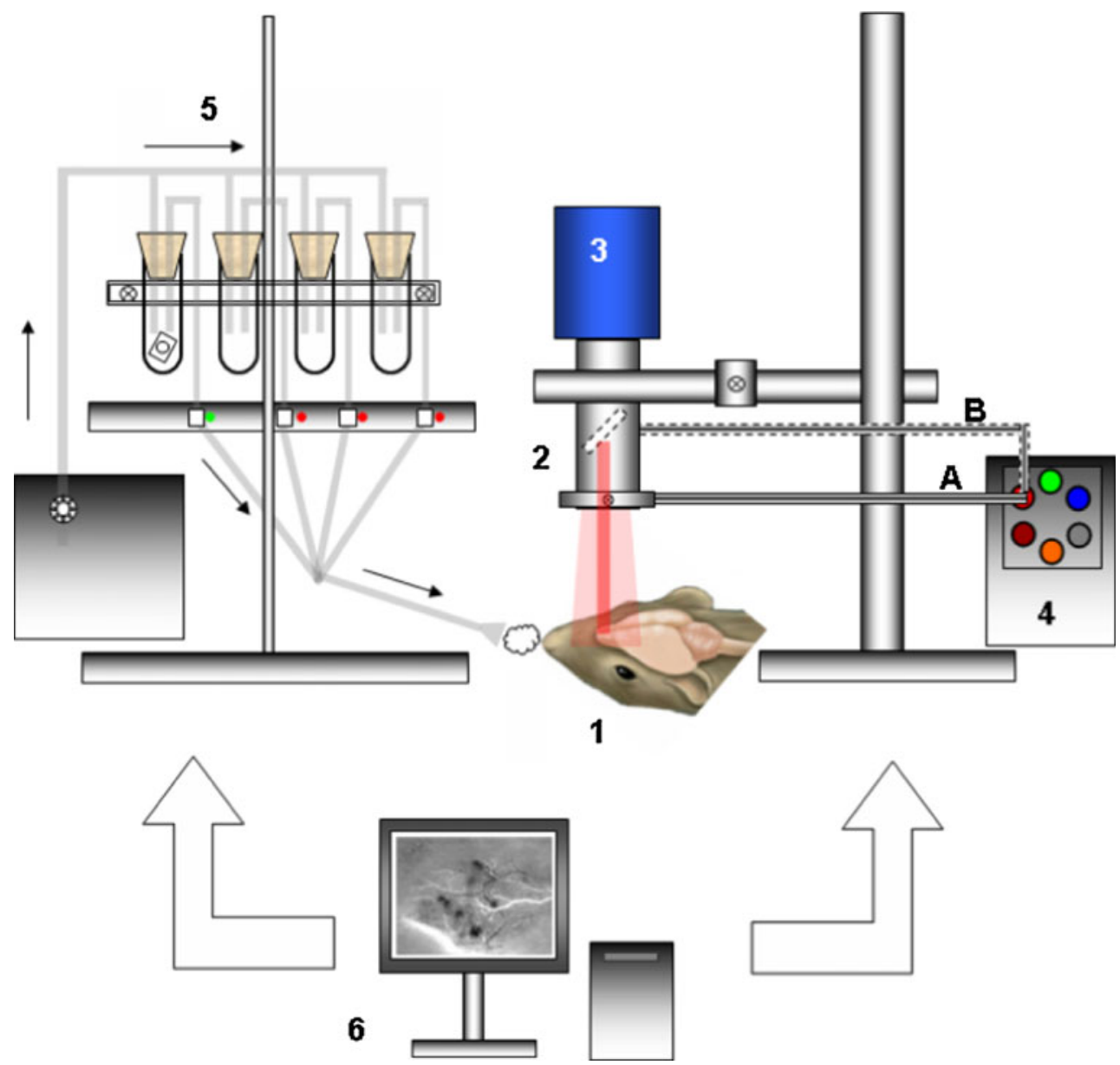

Fig. 4 Wide-field optical imaging set-up for mapping odor-evoked activities in rodents. 1 Anesthetized animal with either full craniotomy or thinned bone above the MOB. Optical window is made of dental cement walls, agarose well and a microscope cover slip. 2 Wide-field microscopy optics with C-mount stereomicroscope or dual lens macroscope. 3 Cooled CCD camera, typically of 12-bit dynamics, with a frame rate of ten images per second or more at full frame. 4 Stabilized white light source with associated filter wheel. Light is shone onto the exposed brain tissue using a goose-neck optical light guide, using an annular fiber ring attached to the optics lens $A$, or through the epi-illumination port of the microscope $B$. Green light is used to visualize the blood vessel architecture. Bandpass interference filters are used to select the light wavelength

for reflectance imaging or excitation of VSD or calcium dyes. For fluorescence studies the emission wavelength is selected using bandpass or long-pass filters placed in the detection path 5. Odors at a given concentration are deposited onto a filter paper and put into sealed vials (here only one odored paper is shown in the left vial in the figure). An olfactometer allows olfactory stimulation control and poststimulation air clean-up. In particular, the use of an air compressor with a manometer and computer-controlled electrovanes allows the mixing of several pure odorants if needed and stimulation for a given intensity and duration. 6 A computer allows the synchronization of illumination, olfactory stimulation and image acquisition. Activation maps are generally processed after acquisition but can also be displayed in video rate mode

drawback of IOS imaging: IOS images of the dorsal MOB allow monitoring of about $20 \%$ of all the glomeruli as opposed to sampling of the entire MOB by fMRI.

What is the biophysical origin of IOS? Early studies of the wavelength-dependence and time-course of IOS were carried out in the cat visual cortex [58]. The intensity of the IOS at different wavelengths followed the absorbance spectra of pure blood and large IOS were recorded for illumination at $570 \mathrm{~nm}$, an isobestic point for the oxy-/deoxyhemoglobin optical absorption spectra. Further comparison of the mapping signal and reflection signal showed that above $590 \mathrm{~nm}$ the reflected signal is much

higher than the mapping signal. This effect was explained by an increased contribution of the oximetric component. In addition, IOS obtained at 570, 600 and $850 \mathrm{~nm}$ show different time-courses, thus justifying the hypothesis of different contribution of the IOS components at each wavelength. The authors proposed the hypothesis that at $570 \mathrm{~nm}$, the signal is purely due to blood volume, whereas above $590 \mathrm{~nm}$ it results from blood volume and oxygenation changes. In addition, the time-course of IOS at $800 \mathrm{~nm}$ is similar to that of scattering signals observed in bloodfree slices. It is likely that the contribution of absorption and scattering changes to IOS differs from one brain area to 
another, since the optical properties of brain tissue depend strongly on the anatomy and physiology of the particular brain area. A study of the change in intrinsic signals versus excitation wavelength was carried out by illuminating the MOB successively at four different wavelengths [45]. The authors reported inadequacies between the magnitude of IOS and absorption modifications expected from changes in local blood volume and oxygen saturation. On the basis of these measurements, they draw the conclusion that at $630 \mathrm{~nm}$ the main source of the signal could be changes in the scattering properties in the GL. However, the authors were uncertain of the cellular basis of these scattering changes. Among several potential sources of light scattering in brain tissue during activation, the more documented are changes in axonal birefringence and cellular swelling, especially from glial cells [59] resulting from ionic exchanges that occur during action potential propagation $[60,61]$ or changes in blood flow [62]. Although several studies in blood-free hippocampus slices have shown the preeminence of scattering changes in the slices [63, 64], to date the absolute contribution of swelling, volume and oxygenation to in vivo IOS remains debated. As a summary, the current hypothesis for the physiological basis of IOS is that the contribution of blood volume effects decreases with increasing wavelength, as oxygenation becomes more preeminent above $600 \mathrm{~nm}$ and scattering becomes non-negligible above $650 \mathrm{~nm}$.

Despite the uncertainty as to the physical basis of the signal, pharmacological experiments have given valuable insights into the cellular mechanisms from which IOS arise [65]. To gain a more detailed view of the origin of IOS in the MOB, the effects of selectively blocking presynaptic dopaminergic and GABAergic receptors, postsynaptic glutamate receptors and astrocytic glutamate transporters were studied. Only the presynaptic glutamate release and uptake by astrocytes were shown to form a critical pathway through which neural activity is linked to functional IOS. These results show that endogenous signals used for functional imaging are induced not at the cell body but locally at the synaptic level [66], and are not linked to postsynaptic spiking activity [34, 67].

\section{Olfactory mapping using activity-dependent exogenous tracers}

Rodents discriminate odors with high accuracy and speed: according to the difficulty of odor discrimination, expert animals respond correctly within 200-350 ms [68, 69]. The initial glomerular stage of information processing thus occurs within $100 \mathrm{~ms}$ of odor presentation. Despite good spatial resolution, metabolic and hemodynamic signals recorded with $2 \mathrm{DG}$ autoradiography, BOLD fMRI or IOS only provide static images of activity patterns representing the whole local neuroglial computation. In addition, neuroenergetic coupling in itself is slower than synaptic transmission. To overcome these limitations and directly map sensory activation in cortices dynamically with higher temporal resolution (dozens of milliseconds time range), a series of new techniques have been developed. In the MOB, activities from presynaptic inputs and postsynaptic dendrites have been recorded in different studies using exogenous fluorescent dyes and have shown temporal dynamics of olfactory maps locked to respiratory rhythm which constitutes the basic temporal processing for odor coding. Remarkably, these techniques allow calcium imaging in awake restrained rats to monitor the effect of sniffing on input activity to olfactory glomeruli. In addition, several interesting studies have compared different optical imaging modalities recorded sequentially in the same animals: this comparison is relatively straightforward since these optical methods share the same recording set-up architecture presented in Fig. 4. These techniques and data are reviewed in the following sections.

Mapping of synaptic potentials using voltage-sensitive
dyes

Characteristics of odor-evoked maps with voltage-sensitive dyes

Voltage sensitive dyes (VSDs) bind to the external surface of membranes of all cells and change their fluorescence intensity linearly with variations in the membrane potential. Changes in dye fluorescence follow those of the membrane potential within a few microseconds, a time constant that is less than the rise time of an action potential, thus allowing imaging with a high temporal resolution locked to the speed of neuronal activity (see Fig. 5 for a comparison of IOS and VSD recordings). Practical use of VSDs has long been hampered in vivo by the weakness of the signal to noise ratio (about $1: 10,000$ or less) due to largely nonspecific staining and absorption of fluorescence photons by hemoglobin. This requires averaging of acquisition frames and long exposure times which Is difficult to perform because of phototoxicity. Hundreds of candidate tracers have been systematically screened in vitro [70], but the development of dyes with excitation wavelengths outside the hemoglobin absorption peak was a breakthrough [71] that led to a notable increase in the signal to noise ratio by about one order of magnitude. Dyes are species-specific and more surprisingly area-specific [72]. Regarding instrumentation, high frame-rate CCD cameras with low intrinsic noise or a photodiode array with high dynamic range have become routinely available allowing VSD imaging; some cameras under development by the 

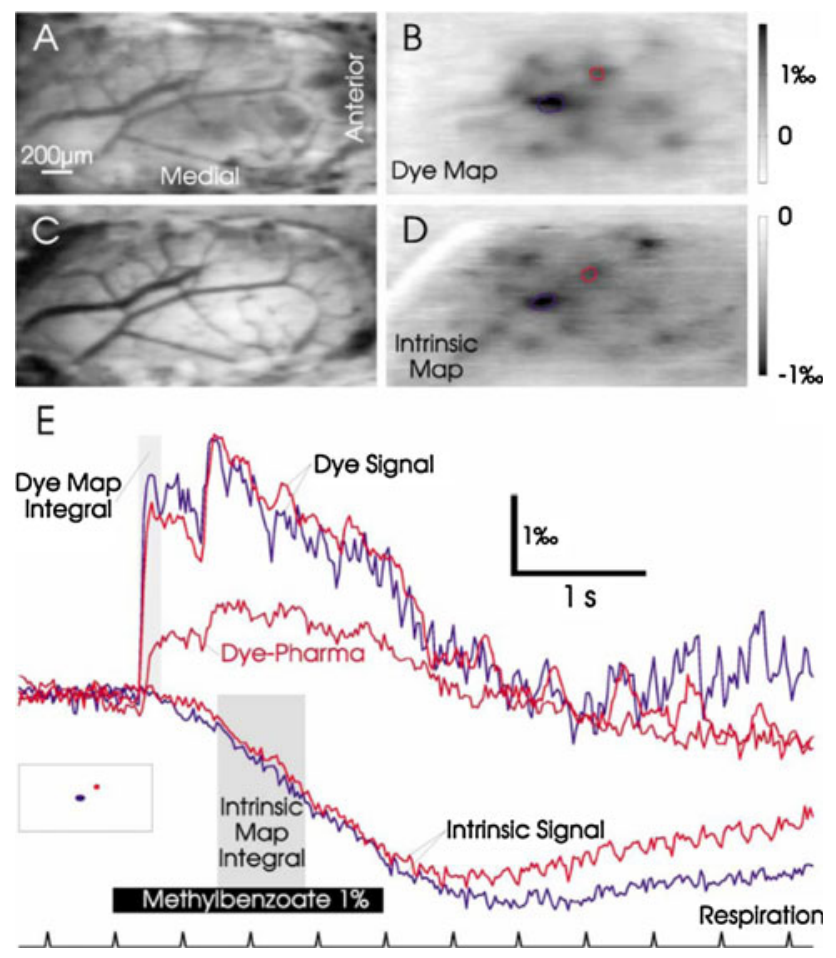

Fig. 5 VSD imaging resolves glomerular activation with high temporal and spatial resolution. a Blood vessel pattern visualized in VSD-loaded dorsal MOB tissues illuminated at $540 \mathrm{~nm}$. b VSD map evoked by presentation of $1 \%$ methylbenzoate. Frames were averaged during the period of the shaded square shown in e. The grayscale (clipping range) $0.08-0.18 \%$ is inverted for comparison with the intrinsic map shown in d. c Blood vessel pattern before intrinsic imaging. d IOS map evoked by the same odor. Frames were averaged during the period of the shaded square shown in $\mathbf{e}$, grayscale $0.12-0 \%$. Maps obtained using the two techniques are very similar although some of the caudal glomeruli did not appear in the VSD map presumably due to poor dye staining. e Reflectance and fluorescence changes plotted as a function of time for the red and the blue regions shown in inset and in $\mathbf{b}$ and $\mathbf{d}$. The VSD signal is significantly faster and locked to inspirations after odor application. IOS peaks after a long delay and does not resolve the respiration-coupled activity. The decline in the VSD signal parallels the beginning of IOS during odor stimulation and may partly reflect contamination of the dye signal by intrinsic optical changes. The trace labeled "Dye-Pharma" shows the time-course for the region with the biggest remaining response after local application of the glutamate receptor antagonists (average of eight repetitions). (Reprinted from reference [72] with permission from Elsevier, copyright 2002)

scientific community are dedicated to in vivo dye experiments (WuTech, http://www.wutech.com/) and complete commercial set-ups are available from Redshirt Imaging (http://www.redshirtimaging.com) and Optical Imaging (http://www.opt-imaging.com).

To our knowledge only two studies carried out by the same group have used this technique in the vertebrate MOB [69, 72] following the first VSD spatiotemporal maps of activity patterns in the MOB obtained in the living salamander by the pioneering studies of Orbach and Cohen [73]. Spors and Grinvald [72] stained the MOB in rats and mice by direct bathing of the tissues with the "blue" dye RH-1838 which gave satisfactory signals (with $\Delta F /$ $F$ intensity up to $4-10,000$ ) but required at least $2 \mathrm{~h}$ of staining after removal of the dura. In this study, activation patterns in the GL were obtained after a single odorant presentation and their spatial and temporal characteristics were studied. Patterns in both rats and mice were odorantspecific and exhibited dynamic sequences of activated groups of glomeruli [72]. As in IOS imaging, the overall amplitude of VSD signals increased with concentration while the latency of the response onset was reduced. Although IOS and VSD maps obtained in the same animals were spatially similar (Fig. 5), they did differ with regard to their temporal dynamics: activated areas had dimensions compatible with individual glomeruli (100-250 $\mu \mathrm{m}$ diameter at half-maximum) but VSD responses had a seven times shorter latency (about $215 \mathrm{~ms}$ to half-maximum) and a rise time about 30 times faster $(\sim 30 \mathrm{~ms})$ than IOS. These fast temporal dynamics allowed a $2 \mathrm{~Hz}$-locked oscillatory pattern in the VSD signal due to respiratory modulation to be shown. In addition, an increase in stimulus intensity led to unchanged maps for the first $40 \mathrm{~ms}$ after the stimulus but later additional glomeruli were activated while the early pattern and sequence were unchanged. Finally, the first odor-induced VSD response was larger than the subsequent ones, which could be explained by presynaptic OSN dynamics or by glomerular processing. Presynaptic calcium imaging (section "Mapping of odor representation at olfactory sensory neurons presynaptic terminals by fluorescent tracers for calcium") further elucidated the temporal dynamics of glomerular activation and when shown together with VSD results, indicates that spatial maps elicited by odors (identity, concentration) show internal temporal dynamics in the activation patterns of individual glomeruli.

\section{Cellular basis of VSD signals}

In a similar manner to IOS, the cellular basis of VSD signals has not been fully described. The population of cells contributing to the changes in fluorescence depends on several experimental factors that are not entirely controlled. The amount of collected fluorescence depends on the staining depth and the extension of the depth of focus of the optical apparatus which might encompass several layers including the ONL, GL and EPL which have different optical properties (absorption and diffusion). The cellular origins of VSD signals are thought to be almost entirely of neuronal rather than glial origin since astrocyte depolarization has a lower amplitude than neuronal depolarization and are delayed by seconds from stimulus onset. Pharmacological experiments in rats [70] have shown that respiration-coupled oscillations at $2 \mathrm{~Hz}$ are blocked by 
tetrodotoxin and diminished by a factor of 2 by ionotropic glutamate receptor antagonists, thus demonstrating that VSD signals arise from the postsynaptic compartment. Given these conditions, it should have been possible to record fast oscillatory activities defined as beta $(15-30 \mathrm{~Hz})$ and gamma $(30-100 \mathrm{~Hz})$, both of these rhythms being related to the reciprocal excitatory inhibitory synapse between mitral and granule cells in the EPL of the MOB. However, the authors failed to observe these oscillations. In their discussion, they suggest that triggering VSD recordings on electrophysiological spike recordings from single identified neurons would help address two issues: (1) the relative contribution of excitatory and inhibitory cells to VSD signals, and (2) the absence fast gamma oscillations in VSD signals [74] which might be generated too deeply to be reached by VSD [75].

Mapping of odor representation at olfactory sensory neuron presynaptic terminals by fluorescent tracers for calcium

Calcium ions are an important target for functional imaging agents because neuronal calcium fluxes are dramatic and directly related to synaptic activity. One widely used method to monitor neural activity relies on the use of calcium tracers which are taken up by cells and located in the cytosol. Three different techniques have been developed to map calcium signals in the MOB: staining of the tissues with a calcium tracer and the use of two models (presynaptic synaptopHluorin $(\mathrm{SpH})$, postsynaptic GCaMPs) of genetically encoded tracers in mice. Interestingly, presynaptic imaging allowed monitoring of OSN input activity to glomeruli during different sniffing frequencies in odor tasks.

\section{Characteristics of odor-evoked maps with calcium dye signals at presynaptic terminals}

In the MOB, a procedure for anterograde labeling of sensory terminals was first used in the zebrafish [76] and later adapted to C57/BL6 mice [77]. Calcium green conjugated with dextran (molecular weight $10 \mathrm{kDa}$ ) is applied to the nostrils and selectively transported to OSN terminals in glomeruli. However, dextran-conjugated dyes are membrane-impermeable, and thus additional treatment of the epithelium with a dilute solution of $0.1 \%$ Triton-X 100, a membrane-permeant agent, is required to allow dye diffusion into OSNs. Using this method, OSN labeling was observed $24 \mathrm{~h}$ after staining and increased in intensity over the next $48 \mathrm{~h}$. Imaging was performed at least 4 days after the staining procedure $[77,78]$. These studies have all been carried out using either the calcium green dextran signals acquired under illumination at $500 \pm 25 \mathrm{~nm}$ with a longpass emission filter with a cut-off frequency at $525-530 \mathrm{~nm}$
$[77,78]$, or the Oregon green Bapta-1 dextran under illumination at $480 \pm 40 \mathrm{~nm}$ with a dichroic filter at $505 \mathrm{~nm}$ and a bandpass emission filter at $535 \pm 40 \mathrm{~nm}$ [79].

Regarding the kinetics of the calcium presynaptic signals, odorants evoked a rapid increase in fluorescence (mean time to half-maximum from 100 to $250 \mathrm{~ms}$ ), much faster than IOS acquired in the same animals (mean time to half-maximum about $1.5 \mathrm{~s}$ ) [80]. After this initial increase, fluorescence signals reach a peak approximately $600 \mathrm{~ms}$ after stimulation onset and rapidly decrease to return to baseline at the end of the stimulation. This rapid decrease seen in about $90 \%$ of glomeruli suggests that calcium signals reflect a true cessation of odorant responsiveness. In OSNs, fluorescence signals are also affected by bleaching and by absorption due to hemodynamic changes [80].

In anesthetized mice, odor-evoked presynaptic calcium maps show strong specificity with the chemical structure of odorant molecules. In addition to spatial patterning of sensory input to the MOB, presynaptic calcium imaging reveals the temporal dynamics of glomerular activation [81]. The time courses of the glomerular calcium signals vary between glomeruli activated by the same odorant, and the spatial map builds up over time with the progressive addition of active spots to the panel of glomeruli. Calcium transients maps involve widely distributed glomeruli and both the number of activated areas and the intensity of the fluorescence response are strongly concentration-dependent $[77,78,80,81]$. Increasing stimulus concentration leads to activated glomeruli in greater numbers and to a large increase in signal amplitude [77, 82]. For hexanal stimulation, the amplitude of the fluorescence variation follows a classical sigmoid dose-response curve and saturates at about $6 \%$ of relative changes, which corresponds to a $10 \%$ odor dilution [78]. Interestingly, increasing sniffing rate artificially through a double tracheotomy induces loss of temporal patterning of odorant-evoked presynaptic calcium signals [81], a mechanism that has been fully described in awake behaving rats.

Wachowiak and Cohen [80] compared calcium imaging signals with IOS at $630 \mathrm{~nm}$ in the same mice. Both signals showed similar stimulus concentration thresholds for different odorants. As can be seen in Fig. 6, the relative amplitude of calcium signals was about three to five times that of the recorded intrinsic signals in the same animals, ranging from $0.7 \%$ to $2.2 \%$ and $0.1 \%$ to $0.9 \%$, respectively, for odorant concentrations increasing from $0.13 \%$ to $5.6 \%$. Interestingly, the dynamic range of calcium signal intensity is larger than for IOS at least in some subsets of glomeruli which leads to similar activation patterns for low-intensity stimulation and significantly different patterns for highintensity stimulation. Activated areas are more focal with calcium signals than IOS with mean full-width at halfmaximum of about 85 versus $122 \mu \mathrm{m}$. Besides these focal 


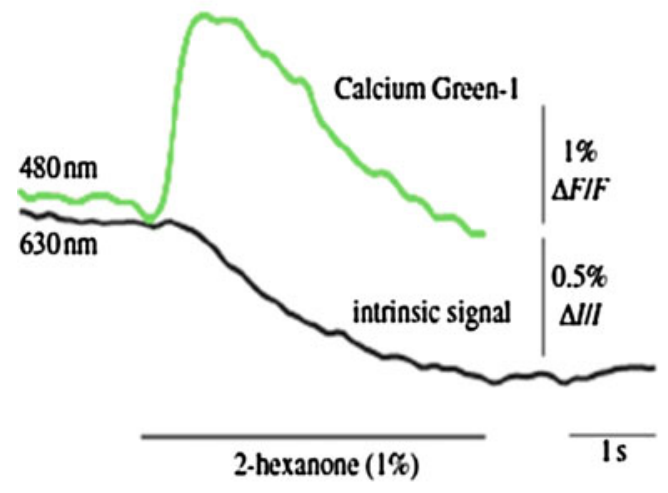

Fig. 6 Comparison of calcium fluorescence maps at the presynaptic level and IOS maps acquired sequentially in the same mice. Left: Time-course and amplitude for each optical signal in a region of interest activated under odorant presentation. Right: Activation maps

activation regions, a general increase in the fluorescence was observed all over the MOB and was attributed to scattered, out of focus light or calcium influx into OSN axons distal to their entrance into glomeruli [77, 78].

\section{High temporal presynaptic calcium imaging in behaving animals: influence of sniffing on activation maps}

Sniffing is a voluntary inhalation of air through the nose that varies in frequency: rodents breath at $<4 \mathrm{~Hz}$ when at rest and can increase respiration to $>4 \mathrm{~Hz}$ with brief bouts of high-frequency sniffing for active odor sampling that can last for only one respiration cycle [83]. High-frequency sniffing is largely expressed during the tracking of an odor source or sampling a new odorant or odors during olfactory tasks, and is thought to be part of the process of odor coding notably by temporally shaping the odor input. The role of these rhythmic sampling behaviors was elegantly studied by Wesson et al. who conducted a series of studies using the high temporal resolution of OSN calcium dyes (down to $8 \mathrm{~ms}$ per image frame) focusing on the glomerular input activity in head-fixed female rats performing a lick/no-lick task in response to odors with simultaneous respiration measurements through a chronic intranasal cannula [84].

As in anesthetized mice [81], temporal patterns of OSNs are diverse in head-fixed rats, and differ among glomeruli and for different odorants and concentrations [79, 85]. The latency and rise-time of sniff-driven OSN inputs are slow, which indicates a progressive activation of OSNs from the same family localized in different regions of the MOE. In low-frequency sniffing regimes recorded in familiar odor discrimination, each inspiration evokes a phasic

\section{Calcium green fluorescence}
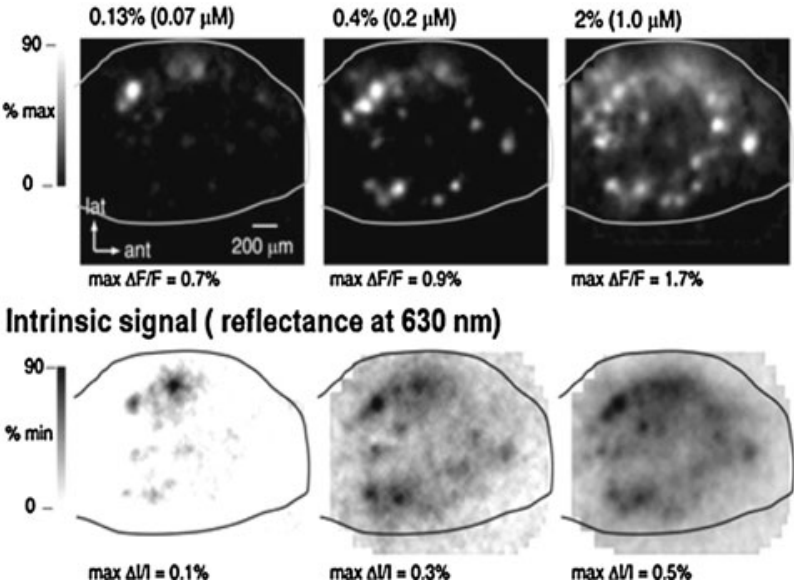

obtained sequentially in the same mice using calcium and intrinsic imaging for increasing stimulus intensities. (Adapted from reference [80], used with permission, copyright 2003))

(100-200 ms) burst of OSN inputs coupled to the sniff cycle (from start of inhalation in one sniff, rise time about $100 \mathrm{~ms}$, time to peak about $150 \mathrm{~ms}$, duration about $400 \mathrm{~ms}$ ), with only a moderate reduction in the amplitude of activated glomeruli over repeated sniffs. At higher sniffing frequencies elicited by novel odor discrimination, calcium glomerular maps for a single odorant remain constant (spatial pattern and amplitude) compared to lower sniffing frequencies [52, 79, 85]. However, phasic input activity is largely reduced over repeated bouts in the presence of the same odorant and is replaced by a tonic one that is uncoupled from the respiratory cycle. This mechanism does not affect the ability of other glomeruli to respond to new odorants encountered later in the sniff bout. Importantly, if a distinct test odor is presented during a high-frequency bout induced for an odorant (knowing that the spatial map of the test odor alone partially overlaps the first one), the resulting calcium map is the subtraction of the two maps taken alone instead of the linear combination that is observed for low-frequency sniffs [79] or odor mixtures [47].

Taken altogether, these mechanisms define an adaptive filter for odor information where the very same stimulus (identity, concentration, duration) can be represented by different spatial maps depending on sniff frequency [79]. This filter would facilitate detection of new odorants in a changing odor landscape by reducing OSN responsiveness to background stimuli. Performance, response times, ability to learn odor associations, or detect and investigate new odorants are the same between head-fixed rats and those engaged in nose-poke-based learning. Interestingly, a major behavior of task rats is their systematic increase in sniffing to $4-10 \mathrm{~Hz}$ during odor sampling $[68,69]$, while head-fixed rats do not change their sniffing regimes when 
performing odor discriminations. In addition, considering that sniffing is also modulated by the auditory discrimination lick/no-lick task, task engagement (odor expectation, movement towards the odor source) might trigger high-frequency sniffing which then would not be important in discriminating one odorant from another. To test this hypothesis, head-fixed rats were submitted to a two-choice odor discrimination task with varied levels of difficulty (binary mixture ratio paradigm or very low odor concentration detection). They performed with high accuracy and showed different sniffing strategies that emerged and were maintained during learning. Some presented brief bouts of rapid sniffing on odorant onset and others none [86]. Thus, high-frequency sniff does not alter odor discrimination or detection threshold, but rather increases the speed of odorant acquisition.

Mapping of synaptic vesicle release from OSN terminals with genetically targeted $\mathrm{SpH}$

Imaging based on a genetically encoded fluorescent protein in the MOB is an elegant alternative to the use of exogenous calcium dyes which might trigger toxicity. Bozza et al. [87] proposed the use of $\mathrm{SpH}$ as a reliable indicator of neurotransmitter release. $\mathrm{SpH}$ comprises a $\mathrm{pH}$-sensitive green fluorescent protein and the mouse synaptic vesicleassociated protein VAMP-2. The fluorescent domain is located inside the vesicles where the $\mathrm{pH}$ is acidic, leading to a low fluorescence level in the basal state. Following neuronal activation of $\mathrm{SpH}$-expressing neurons, the vesicle docks at the presynaptic terminal plasma and its lumen becomes continuous with the extracellular space, leading to an increase in $\mathrm{pH}$ and a subsequent increase in fluorescence. A mouse strain with all OSNs expressing $\mathrm{SpH}$ was created using an olfactory marker protein (OMP, selectively expressed in OSNs) tag in embryonic stem cells. The fluorescence observed in glomeruli is due to some of the $\mathrm{SpH}$ located in the plasma membrane. Among glomeruli this intensity varies because of the difference in OMP expression within OSNs. These mice are currently commercially available from the Jackson Laboratory (Bar Harbor, ME; stock \#4946).

\section{Characteristics of odor-evoked SpH fluorescence maps}

Odorant presentation elicits increases in focal fluorescence in OMP-SpH mice that reliably indicates the amount of transmitter release from OSN terminals [82, 88]. The average diameter of the focally activated areas is about $93 \mu \mathrm{m}$ and is considered as a single activated glomerulus [87]. Bleaching is quantified as a continuous exponential decrease with a time constant of about $2 \mathrm{~s}$ and a typical decrease of $6-7 \%$ of baseline fluorescence intensity for a 10-s illumination [89]. Fluorescence levels recover almost entirely after $90 \mathrm{~s}$. Bleaching is generally corrected through frame-by-frame subtraction of a no-odor trial image acquired just before the odor stimulation trial. As for other optical techniques, $\mathrm{SpH}$ maps are odorant-specific, relatively similar for an odorant across preparations and roughly symmetrically bilateral [87]. Experiments with saturation vapor between $0.1 \%$ and $1 \%$ for several odorant stimuli led to relative changes of fluorescence up to $4 \%$ [51]. The $\mathrm{SpH}$ signals varied differently with increasing odorant concentration across glomeruli in the same mouse: for hexanone odorant, $0.1-10 \%$ of the glomeruli showed saturation for concentrations above $1 \%$, whereas others did not saturate. Surprisingly, some glomeruli exhibit a nonmonotonic dose-response curve, where signal amplitudes increase for concentrations up to $0.3 \%$ and then decrease to a plateau for concentrations above $1 \%$. This variation can be explained by OSN adaptation at high concentrations or presynaptic inhibition of transmitter release during highfrequency action potentials [87].

$\mathrm{SpH}$ signals show complex temporal kinetics. In contrast to calcium fluorescence signals, they show a slow onset after odor presentation and a long recovery. A linear model for $\mathrm{SpH}$ signals up to their maximum was proposed considering four components: resting fluorescence, exponential decrease due to bleaching, a transient decrease in fluorescence immediately after stimulation, and odorevoked rise in fluorescence $[82,88]$.

No finely organized mapping of carbon chain length was observed in initial studies using $\mathrm{SpH}$ signals [87] in contrast to former IOS or 2DG imaging [24, 46].

\section{Comparison with other optical imaging signals}

The influence of hemodynamic signals on $\mathrm{SpH}$ fluorescence has been assessed in initial studies [87]. In mice with OSNs anterogradely labeled with Alexa Fluor 488 (whose fluorescence is independent of activation), Bozza et al. recorded a slow decrease in fluorescence after odorant stimulation. Since the decrease was markedly stronger above blood vessels, it was attributed to an increase in absorption of fluorescence photons due to hemodynamics producing IOS. The amplitude of $\mathrm{SpH}$ fluorescence decreased by $50 \%$. The authors concluded that IOS contamination could distort the time-course and amplitude of the $\mathrm{SpH}$ signals, but should not alter qualitatively the response patterns. More recently, $\mathrm{SpH}$ signals were directly compared with IOS in the same animals using interleaved illumination at 470 and $780 \mathrm{~nm}$ [51]. Both signals showed the same odor dependence. The amplitude of $\mathrm{SpH}$ signals and IOS were recorded for two glomeruli for an extensive 
set of 88 odorants. The relative changes in both signals varied linearly in respect to each other, but the slope of the linear relationship was different for the two glomeruli. Notably the amplitude of IOS was about 10-20 times lower than that of $\mathrm{SpH}$ signals (maximum response amplitude of $0.15 \%$ vs. $3 \%$ ). In addition, even adjacent glomeruli could be better resolved spatially with $\mathrm{SpH}$ signals than with IOS.

$\mathrm{SpH}$ signals have been compared with presynaptic calcium signals in OMP-SpH mice using anterograde labeling of OSNs with calcium-sensitive rhod dextran [87, 88]. This tracer has a low affinity for calcium channels, and consequently minimally disturbs the synaptic exocytosis and leaves the $\mathrm{SpH}$ signal amplitude and temporal characteristics unchanged. Comparison of $\mathrm{SpH}$ and rhod dextran signals in the same animals has shown that more glomeruli show $\mathrm{SpH}$ signals: about four of five glomeruli showing rhod signals also show SpH signals, while only one of three glomeruli showing $\mathrm{SpH}$ signals also show rhod signals. Incomplete dye loading or lower sensitivity of rhod might explain this discrepancy. Furthermore, the correlation between the amplitudes of $\mathrm{SpH}$ and rhod signals in vivo is weak which can be attributed to improper dye loading, dye saturation and potential nonlinearities between presynaptic calcium influx and transmitter release [87]. The relationship between extracellular calcium concentration and $\mathrm{SpH}$ signals was studied further in slices and showed a superlinear relationship, implying that a moderate change in presynaptic calcium influx strongly modulates the amount of neurotransmitter released from OSNs [88]. Finally, there is a monotonic linear relationship between odor-evoked $\mathrm{SpH}$ fluorescence and presynaptic spiking [15], indicating that this signal reliably tracks input activity to the recorded glomerulus (Fig. 7).

\section{Cellular basis of OSN presynaptic signals}

Presynaptic calcium signals are strongly linked to afferent spiking activity on presynaptic axonal terminals [90]. This
Fig. 7 Presynaptic imaging of $\mathrm{SpH}$ for olfactory mapping. a, b Confocal microscopy images of the surface of MOB slices for comparison of fluorescence resulting from anterograde calcium labeling and $\mathrm{SpH}$. Labeling of axon bundles in the ONL and GL are seen with Alexa Fluor dextran, whereas only glomeruli are labeled in $\mathrm{SpH}$ mice. For both labelings, no fluorescence is seen in the periglomerular area. c Comparison of the amplitude and time-course of calcium (high-affinity rhod tracer) and $\mathrm{SpH}$ signals recorded alternately in the same glomerulus. Both traces are from a single trial. The onsets of the signals are the same following stimulation, but the rise time of the rhod signal is much shorter than that of $\mathrm{SpH}$ (amplitude bar $\Delta F / F$ : $\mathrm{SpH} 1 \%$, rhod $0.2 \%$ ). d Resting fluorescence in $\mathrm{SpH}$ mice. e Activation maps induced by a 2-s presentation of $1 \%$ hexanone shows bilaterally symmetrical maps.

(a-c Adapted from reference [88] used with permission; d, e reprinted from reference [87] with permission from Elsevier, copyright 2003)
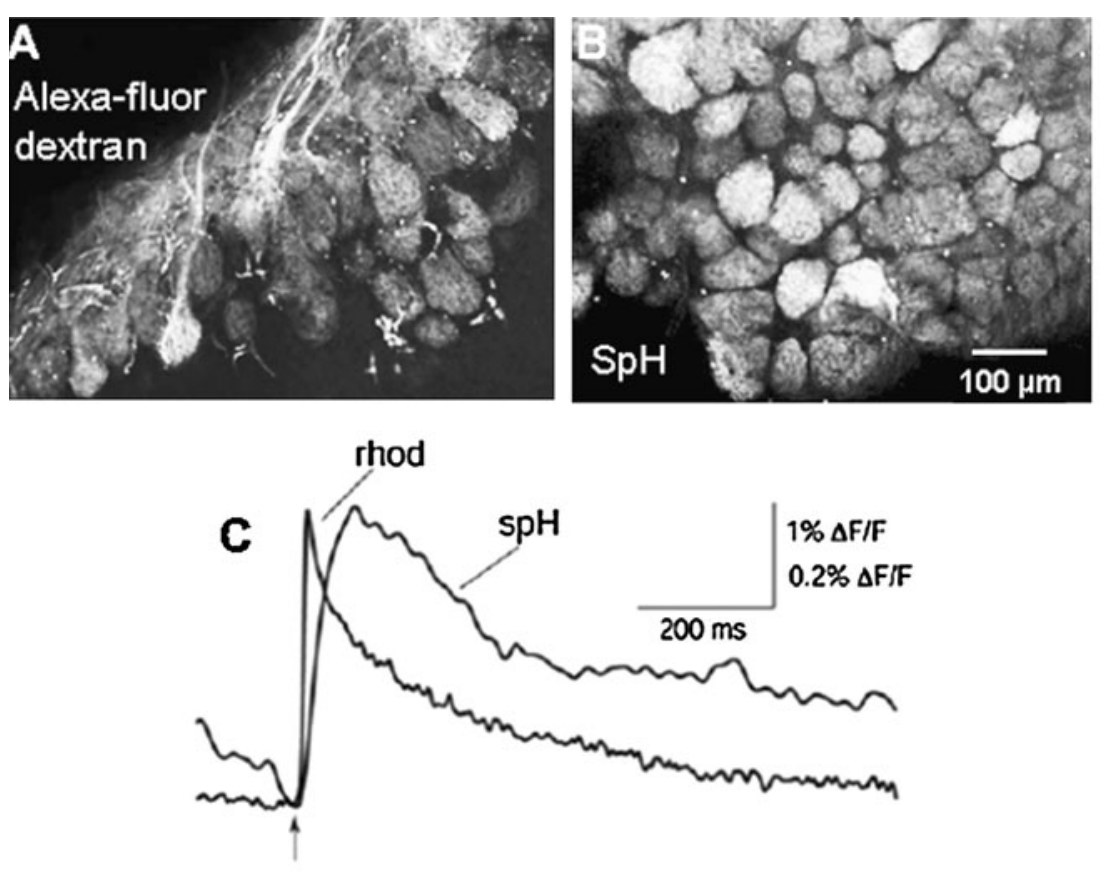

resting fluorescence

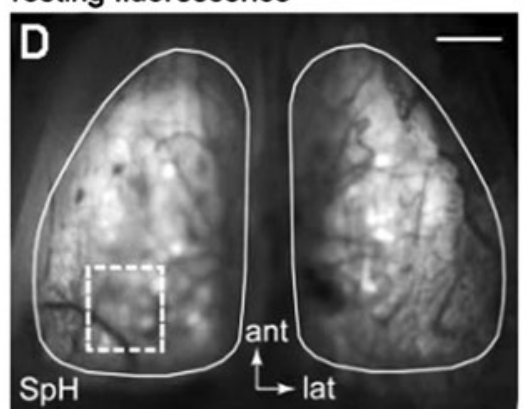

$1 \%$ hexanone $(4.7 \mu \mathrm{M})$

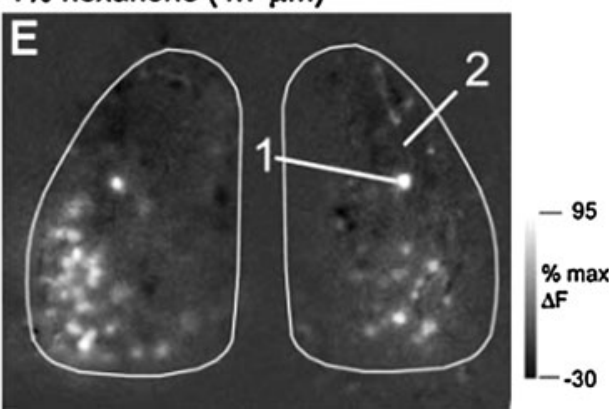


relationship can be modulated by local inhibition since presynaptic OSN terminals express gamma-aminobutyric acid B (GABA-B) receptors that provide a potent local inhibitory input [91] which might result in a center-surround inhibition described for other sensory circuits. Vucinić et al. [92] tested this hypothesis using both calcium green-1 dextran fluorescence and GABA-B receptor pharmacology. They showed that strongly activated glomeruli (center) appear to suppress the release from receptor neurons terminating in surrounding glomeruli. Contrary to these results, McGann et al. [82], using $\mathrm{SpH}$ mice with GABA-B receptor pharmacology, observed that presynaptic inhibition strongly modulated the amplitude of $\mathrm{SpH}$ signals within a glomerulus (probably to prevent postsynaptic cells from saturation for high-intensity stimuli) while the relative spatial pattern on the MOB remained unchanged. This discrepancy can be explained by the molecular basis of both fluorescent signals in vivo (fluorescent dye versus genetically encoded indicator) as shown in the previous section. Further details on the effects of GABA-B inhibition on OSN presynaptic terminals were elegantly and definitively described by Pírez and Wachowiak [93] who used the same protocol as Vucinić et al. [92]. These authors showed that in addition to a mild phasic effect, an important tonic regulation is mediated by GABA-B receptors on odor-evoked presynaptic calcium signals in the mouse glomeruli. This tonic inhibition takes place in all glomeruli and does not depend on the strength of OSN inputs nor the sniffing frequency used to sample the odors. In addition, no center-surround inhibition could be found using the GABA-B receptor pharmacology. Thus, presynaptic tonic GABAergic inhibition in the mouse olfactory glomeruli does not seem to be primarily involved in the gain control of OSN inputs. This results in a balanced presynaptic modulation that varies according to the strength of presynaptic inputs. An alternative purpose of GABAergic inhibition may be to serve as the effector through which the centrifugal fibers can modulate OSN inputs [94]. It has to be noted that these findings do not exclude the possibility that phasic GABAergic release could mediate an intraglomerular gain control of synaptic inputs nor that short axon cells could be responsible for interglomerular lateral interactions [95] since they interact with PGC-M/TC synapses rather than presynaptic PGC-OSN ones.

More recently, the influence of centrifugal serotoninergic modulation on odor input was studied in vivo using wide-field two-photon microscopy in $\mathrm{SpH}$ mice [94] and the pharmacology of the serotoninergic receptors. The authors demonstrated that 5-HT2C receptors located on PGCs mediate inhibition of odor-evoked $\mathrm{SpH}$ response via GABA release.
Optical imaging of odor-evoked maps at the postsynaptic level in genetically engineered GCaMP2 mice

Glomerular postsynaptic activity has been successfully isolated with an optical imaging approach using a transgenic mouse expressing a genetically encoded calcium indicator, the calcium-sensitive green fluorescence protein (GCaMP2) under the Kv3.1 potassium channel promoter $[96,97]$. Wide-field imaging of odor-evoked maps in these transgenic mice has been carried out by Fletcher et al. [98]. Immunohistochemistry characterization of $\mathrm{MOB}$ cells expressing GCaMP2 showed a strong labeling of M/TCs and PGCs including the external tufted cells within the GL $[98,99]$. Fluorescence was excited at $480 \mathrm{~nm}$ and collected with a band-pass filter at $535 \pm 50 \mathrm{~nm}$ and recorded through an epifluorescence microscope with a back illuminated CCD camera with high sensitivity and low noise which allowed a frame rate of $25 \mathrm{~Hz}$. Resting fluorescence in GCaMP2 mice was diffuse and its amplitude about $60 \%$ higher than in wild-type mice [98].

\section{Characteristics of odor-evoked fluorescence maps in GCaMP2 mice}

Odor presentation induces two types of fluorescence signals: spatially diffuse signals of low amplitude, and focal increases of high amplitude. Focal increases were to some extent symmetrically spread over the two MOBs with typical amplitudes of about 4-6\% under a $0.25 \%$ saturated vapor of butyraldehyde presented for $4 \mathrm{~s}$ [98] (Fig. 8). The decrease in fluorescence due to bleaching was about $8.5 \%$ in $20 \mathrm{~s}$ which was corrected using either frame-by-frame subtraction of a no-odor trial image or exponential fitting of the fluorescence decay prior to odor stimulation. The mean full-width at half-maximum of focal spots was $67 \mu \mathrm{m}$ which was similar to that of glomeruli identified from resting fluorescence in $\mathrm{SpH}$ mice [98]. Postsynaptic odorevoked maps were acquired first for a set of odorants with distinct and similar molecular features, and second for increasing saturated vapor concentration of pentanal ranging from $0.06 \%$ to $10 \%$. Odor-evoked maps were odorantspecific and showed coarse chemotopic organization. Increasing concentrations led to recruitment of additional glomeruli and increases in the GCaMP2 signal amplitude. About $32 \%$ of all activated glomeruli were already activated with $0.06 \%$ pentanal but the response of some glomeruli did not show evident saturation at $10 \%$. The dose response function was fitted to a Hill function and led to values similar to those obtained with presynaptic calcium mapping [77].

Temporal dynamics of GCaMP2 maps are slower than presynaptic calcium signals obtained with a mean rise time 

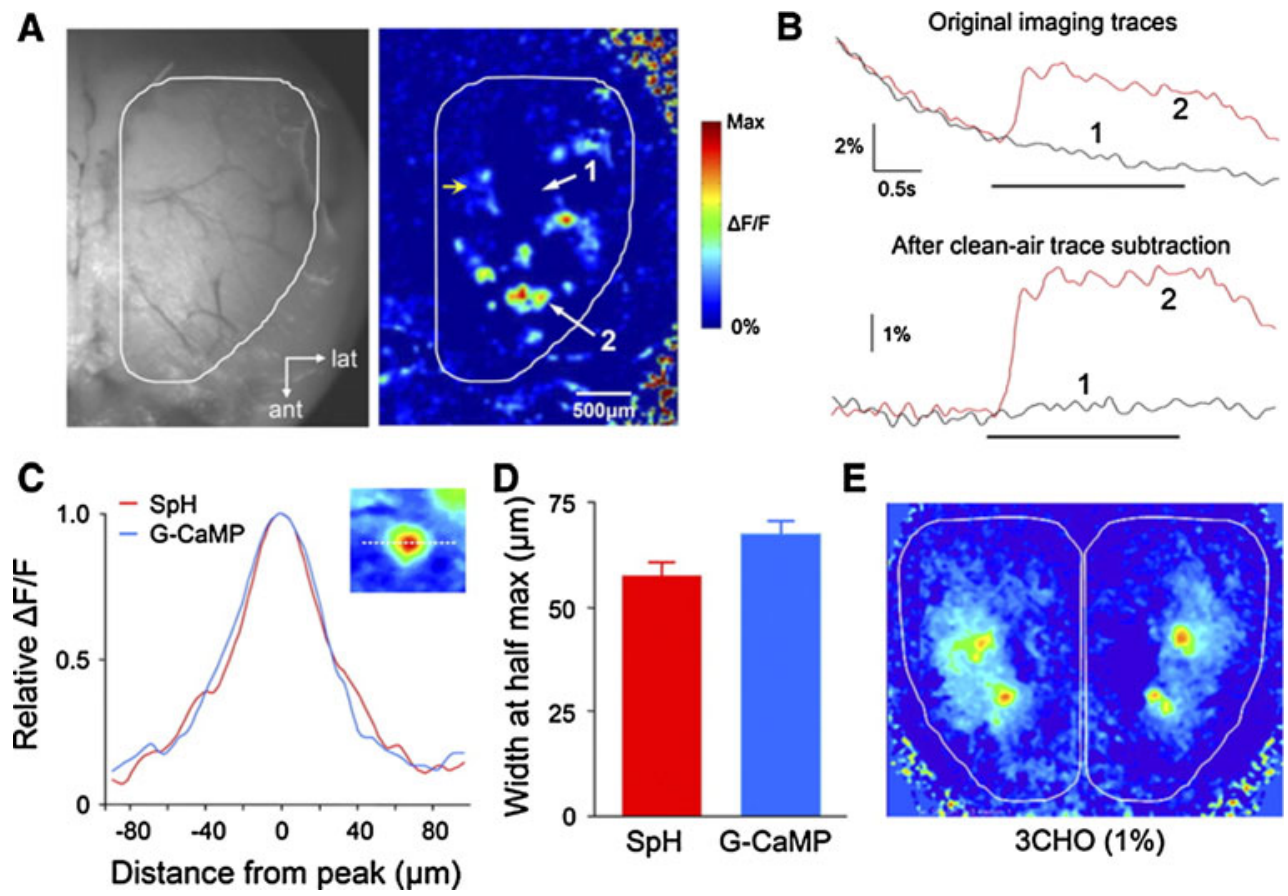

Fig. 8 Odorant-evoked GCaMP2 calcium responses in the MOB. a Left: Resting fluorescence image of the MOB surface observed through the thinned skull of a GCaMP2 mouse. Right: A single-trial odor response map induced by butyraldehyde $(0.25 \%$ of saturated vapor). Arrows indicate the locations where the odor-evoked response traces shown in b were obtained. b Top: Raw fluorescence traces without photobleaching correction. The red and gray traces are the GCaMP2 signals measured, respectively, in odor-activated (trace 2) and nonactivated regions (trace 1) as indicated in a. The black

of about $241 \pm 80 \mathrm{~ms}$. As for calcium presynaptic signals, odor-evoked maps are elicited after the first sniff during odorant presentation. For some glomeruli there is a respiration-coupled phasic decrease in recorded fluorescent signals. This modulation was more marked for mediumintensity stimulation than for high-intensity stimulation, and could not be separated from noise for low-intensity stimulations.

\section{Cellular basis of GCaMP2 signals}

The pharmacology of GCaMP2 fluorescence has been characterized both in vitro and in vivo but only for electrical stimulation which only moderately mimics odorevoked activation. Topical application of ionotropic and metabotropic glutamate receptor antagonists in vivo suppressed these electrical activations, thus confirming the postsynaptic origin of GCaMP2 signals [98].

Functional MRI with manganese as a contrast source

Manganese-enhanced MRI (MEMRI) allows mapping of neuronal function and connections (for a review see horizontal bar indicates a 2-s odor delivery. Bottom: Same odor responses with photobleaching subtraction using a no-odor imaging trial. c Comparison of the averaged $\Delta F / F$ response spatial profile in odor-evoked hot spots in the GCaMP2 mice (blue) and from $\mathrm{SpH}-$ labeled olfactory glomeruli in the OMP-SpH mice (red). d Quantitative comparison of the mean widths measured at half-maximum in the GCaMP2 and OMP-SpH mice. e Odor-evoked GCaMP2 response maps are bilaterally symmetrical (adapted from reference [98], used with permission)

reference [100]). Manganese is a paramagnetic calcium mimetic and accumulates in cells in an activity-dependent manner entering through voltage-gated calcium channels. It is transported anterogradely along axons and can cross synapses. Manganese has an life-time of 2-3 weeks after a single injection. Pharmacological experiments using glutamate receptor pharmacology have shown that the magnesium-dependent signal enhancement results from the activation of glutamatergic neurons through NMDA ( $N$-methyl-D-aspartate) receptors, but not through AMPA (alpha-amino-3-hydroxy-5-methyl-4-isoxazolepropionic acid) receptors [101]. Odor-induced activity recordings with functional MEMRI were first demonstrated by Pautler and Koretsky [102]. MEMRI signals in the MOB can be obtained either by intraperitoneal injection of manganese chloride (which might induce neurotoxicity [103]) or by nasal injection which is the preferred pathway to map the connectivity in the olfactory system. Recently, functional maps of odorant stimulation with individual glomerular spatial resolution were obtained [104]. After injection of $7 \mu 10 \mathrm{mM}$ manganese chloride into each nostril, MEMRI maps were acquired using $\mathrm{T} 1$-weighted contrast under a 11.7- $\mathrm{T}$ magnet with a $0.01 \mu \mathrm{m}$ spatial resolution and 20 min temporal resolution. After sustained odor exposure 
over $20 \mathrm{~min}$, MEMRI maps showed odor-specific and bilaterally symmetrical enhancement in the outer layers of the bulb that were similar across animals for the same odorant. One unique aspect of the technique is that it theoretically allows information flow to be followed in the olfactory network from the MOE to the cortex, since manganese can be transported through active synapses from the glomeruli down to M/TCs and the olfactory cortex [102]. However, this promising technique is limited to static imaging, as is 2DG autoradiography, and cannot provide information on neural dynamics due to the slow accumulation of manganese.

\section{Towards new functional optical imaging of olfactory glomeruli}

Functional imaging techniques are constantly being developed at a high pace driven by challenging applications in the preclinical and clinical fields. One of the key technologies that has emerged in the last decade for in vivo studies is two-photon scanning laser microscopy (TPSLM) [105]. This technique provides cellular resolution and can be implemented with fluorescence-based microscopy techniques such as life-time fluorescence imaging, fluorescence resonance energy transfer, photostimulation or active molecules uncaging. The technique relies on the fast scanning of the tissues with femtosecond lasers tunable in the infrared wavelengths and focused at the plane of interest where nonlinear processes are elicited, leading to local fluorescence excitation. This optical sectioning mechanism allows elimination of out of plane fluorescence, while the use of infrared wavelengths provides deeper penetration and less phototoxicity.

For the study of the MOB, TPSLM benefits from the low depth of the GL that largely limits light scattering effects in tissues. In recent years many in vivo studies of MOB activation at the cellular level have used TPSLM in conjunction with extrinsic fluorescent calcium tracers in normal [106, 107] and OMP-SpH mice [15, 92]. Very recently, TPSLM and phosphorescence quenching were combined with an optimized oxygen nanoprobe sensor [108] for high-resolution mapping of oxygen partial pressure in the rat cortex [109]. Another field where TPSLM has proven particularly relevant is the study of blood flow changes during activation [110]. Quantitative measurements of local hemodynamics in the GL have been obtained in several studies by Tiret et al. at the capillary level and have been reviewed recently [111]. After injection of a fluorescent molecule in plasma through the femoral vein, images of the glomeruli show that capillaries are scarce in the ONL and mainly located in the GL and below [112]. A rapid longitudinal line scan of capillaries (about $15 \mu \mathrm{m}$ in $1 \mathrm{~ms}$ ) shows tracks of red blood cells (RBCs) as shadows in fluorescent plasma. This allows measurements of RBC instantaneous flow, velocity, linear density and longitudinal elongation in individual capillaries. Odor presentation elicits increases in RBC flow which is associated with increases in their velocity $(1.2 \%$ hexanal presentation led to more than $40 \%$ increase in $\mathrm{RBC}$ velocity [13]). Measurements in two neighboring glomeruli have shown that vascular responses seem glomerulus-specific [13]. However, the need to perform a fast line scan limits the field of view of the technique and does not allow exhaustive studies of all adjacent glomeruli [113]. Another limitation arises from the limited number of moving RBCs at a given location immediately after stimulation, which impairs measurements of vascular changes in the first hundreds of milliseconds after stimulation. Simultaneous localization of capillaries and glomeruli can be achieved after labeling of OSN inputs to the MOB with calcium Oregon green [15].

Two-photon microscopy is by construction devoted to a small field of view that encompasses only a few adjacent glomeruli (five in reference [113]) in the case of the MOB. In this context, mapping of activation-related blood flow changes over a large field of view can be achieved with scanning laser Doppler imaging which relies on the shift in wavelength of photons reflected by moving scatterers. The technique allows the mapping of relative flow changes in the somatosensory cortex [114, 115], but has a coarse spatial resolution (at least $1 \mathrm{~mm}^{3}$ ) which has limited interest in it for glomerular activation mapping. The implementation of full-field laser speckle imaging has been proposed to overcome the limitations of laser Doppler imaging [116, 117]. Laser speckle is an interference pattern observed by the coherent addition of scattered laser light with slightly different path lengths. When an area illuminated by laser light is imaged with a camera, a granular pattern named speckle is produced. If the scattering particles are moving, the speckle pattern at each pixel varies with time. The temporal and spatial intensity variations of this pattern contain information about the motion of the scattering particles. In particular, computing the changes in the local speckle contrast in the image indirectly provides a measure of relative changes in blood flow, since the speckle contrast is related to the movements of the scatterers: the faster the flow, the less contrast in the recorded pattern. Several groups have used the spatial [118-122] and temporal contrast [123] to map relative blood flow changes in the somatosensory cortex, but the technique has not yet been applied to the MOB. One challenge for laser speckle imaging is the development of accurate models for speckle pattern formations in the context of blood flow measurement to provide absolute quantitative measurement of flow changes [124]. Ultimately, laser Doppler imaging and laser 
speckle imaging share the limitation of being $2 \mathrm{D}$ techniques that cannot record deep optical changes, which is probably less critical for GL activation due its superficial location as compared, for example, to layer IV in the cortex.

Optical coherent tomography (OCT) could address this drawback in the near future. It relies on the detection of back-scattered light from the tissues illuminated with a low temporal coherence source in a Michelson interferometer configuration, where a light beam is split into a reference arm and a measurement arm. Reflected light from both arms forms an interference pattern which contains information on the path traveled by the light in the measurement arm. OCT uses illumination in the infrared region with a broad spectrum that implies that interference occurs only for photons whose path lengths are very close. This provides a physical optical sectioning mechanism. Modifying the length of the reference arm allows recording of OCT signals at different depths and provides coronal images down to $2 \mathrm{~mm}$ below the surface. Depth-resolved measurements in the activated brain have been obtained in the cat visual cortex [125] and the rat somatosensory cortex [126]. The general assumption regarding the origin of functional OCT (fOCT) contrast is that it arises from changes in the scattering properties of the tissue; however, the physiological origins of these signals remain debated. Initial studies have attributed fOCT signals to local structural changes such as capillary dilation and cell swelling. Implementation of Doppler OCT [127-129] and a refined variant called optical microangiography [130] have allowed depth-resolved measurements of absolute blood flow in the rat brain with high spatial resolution and temporal resolution compatible with the study of hemodynamics. Applications of fOCT to MOB studies present a very exciting technical prospect.

\section{Conclusion and future directions}

After the "olfactory revolution" in the 1990s, the structural properties of olfactory wiring between the OSNs and olfactory glomeruli were described. Beyond the spatial limitations of classical tools such as electrophysiology, several recent functional imaging modalities using signals from endogenous and exogenous sources have made possible the functional description of the spatial distribution of activity among olfactory glomeruli. Calcium dyes and VSDs have also provided some information about the temporal dynamics of these activation maps with respect to breathing and sniffing rhythms.

We conclude this review with a summary of the main discoveries concerning the odor-evoked spatial patterns of activity across the olfactory glomeruli resulting from functional imaging studies and the current open questions about the relative odor sensitivity and diversity of olfactory glomeruli and mitral cells.

Spatial distribution of odor-induced activities in the MOB: the question of glomerular chemotopy

Despite differences in the rodent species, anesthesia regimes, odor presentation (identity, concentration, and duration), recording set-ups, and above all, origins of the functional signals, all the functional imaging techniques that we describe in this review have repeatedly provided a striking picture of how odor identity and concentration are represented by a spatial code in the MOB. We emphasize in particular the technical issues for each imaging procedure, describing in detail the biophysics of functional signals and comparing spatiotemporal resolution between imaging modalities. The overall odor picture that we can depict in the MOB from these data across rodents is as follows: odorants with different functional groups activate distinct domains; increasing odor concentrations reduce response latencies, increase response amplitudes, and recruit new glomerular units in wider areas; and enantiomers are represented by overlapping but distinct space maps and can be distinguished by rodents in olfactory tasks. Although the issue of center-surround inhibition mechanisms seems to be conclusively absent across olfactory glomeruli, whether incremental increases in chain length of aldehydes and alcohols may result in a gradual (although discontinuous) anterolateral shift of activated areas in the MOB remains an open question. Interestingly both metabolic (2DG) and hemodynamic (IOS, BOLD) signal imaging have detected this shift in the MOB space, while exogenous dyes (calcium and VSDs) have failed to do so. However, carbon chain length is one of many dimensions of a molecular structure that can vary, and other properties might be involved in this spatial representation as neighboring glomeruli do not necessarily code for the same odorant feature [51].

Temporal dynamics of odor-induced activities in the MOB: the breathing and sniffing drive

Odorant sampling is constantly changing during natural odor-guided behaviors in which the odor environment, and hence sniffing behavior, is complex and temporally dynamic. Respiration-synchronous $2-\mathrm{Hz}$ patterns of glomerular activation were shown in anesthetized rats by VSD imaging. In awake head-fixed rats expressing odor-guided behaviors undergoing presynaptic calcium imaging, high frequency sniffing is unexpectedly not involved in information processing and not necessary for simple odor 
discrimination tasks, but rather facilitates odor acquisition. Further exploration of the temporal dynamics of glomerular input (OSNs) combined with the monitoring of their output (M/TCs) and their relationship to respiratory cycles is an exciting prospect for these studies. It is important to note that the main characteristic features of MOB networks were determined in slices by delivering single or repeated ONL shocks (mimicking natural sniffing) to drive M/TCs. These data have to be carefully considered because they were obtained without the slow recruitment and rapid termination of OSN input activity observed in vivo and the interleaved low- and high-frequency sniffing patterns in awake rodents. Finally, the precise role of centrifugal feedback to the MOB [131] in the temporal shaping of odors remains to be explored.

Sparse codes in the MOB

Until recently mitral cell activity was thought to arise from its excitation by the glomerulus where it projects combined with the broad inhibitory inputs from the large surrounding field of the glomerulus. This mechanism was thought to precisely shape an olfactory column by the inhibitory effect of the most active glomeruli/mitral cells on the weaker surrounding ones. This was supported both by extensive anatomical data showing a very well-defined hard-wired framework of glomeruli and by extensive and dense lateral interactions between mitral and granule cells. In addition, an elegant approach combining IOS and intracellular recordings of PGCs, M/TCs and granule cells have supported this view [48]. However, using IOS combined with many extracellular recordings of mitral cells (defined by their firing and their phase with respect to breathing) and with a larger panel of odors, Fantana et al. [132] showed that in addition to the central glomerulus that excites the mitral cell, only a small subset of glomeruli actually inhibit its activity. Very surprisingly, these lateral glomeruli do not respond to similar chemical structures and are distant in space in the MOB. Thus, like glomerular activation [93], mitral cells do not seem to present center-surround receptive fields. These data contradict the lateral inhibition concept that has dominated from the 1980s to the present day. Alternative explanations exist and encompass the view of lateral interactions, binding large ensembles of mitral cells together instead of contrasting coupled ones [133]. Clearly, further studies are needed to question the actual existence of lateral inhibition at the glomerular and mitral/ granule cell level.

Finally, Soucy et al. [51] recently demonstrated that no chemotopic order is apparent on a finer scale, and nearby glomeruli are almost as diverse in their odor sensitivity as distant ones. So taken together with [132] these data, the burning question is how to get a sharp and meaningful output from such loose (coarse, sparse, diverse) odor activation patterns in glomeruli. To solve this crucial issue, the precise roles of glomerular cellular populations such as the external tufted cells [99, 134], capable of temporally coordinating glomerular outputs with a lock on sniffing frequencies, have to be further explored in vivo. To this end, exciting and challenging technical combination of optical imaging, molecular biology and electrophysiology are needed in awake animals performing behavioral tasks.

Acknowledgments The authors gratefully acknowledge Claire Martin and Brenda Chen for insightful comments on the manuscript. Work in our laboratory is supported by Agence Nationale de la Recherche ANR-09-JCJC-0117-01.

\section{References}

1. Mombaerts P (2004) Genes and ligands for odorant, vomeronasal and taste receptors. Nat Rev Neurosci 5:263-278

2. Friedrich RW (2006) Mechanisms of odor discrimination: neurophysiological and behavioral approaches. Trends Neurosci 29:40-47

3. Kay LM, Beshel J, Brea J, Martin C, Rojas-Líbano D, Kopell N (2009) Olfactory oscillations: the what, how and what for. Trends Neurosci 32:207-214

4. Galizia CG, Rössler W (2010) Parallel olfactory systems in insects: anatomy and function. Annu Rev Entomol 55:399-420

5. Adrian ED (1950) The electrical activity of the mammalian olfactory bulb. Electroencephalogr Clin Neurophysiol 2:377-388

6. Buck L, Axel R (1991) A novel multigene family may encode odorant receptors: a molecular basis for odor recognition. Cell 65:175-187

7. Vassar R, Chao SK, Sitcheran R, Nuñez JM, Vosshall LB, Axel $\mathrm{R}$ (1994) Topographic organization of sensory projections to the olfactory bulb. Cell 79:981-991

8. Ressler KJ, Sullivan SL, Buck LB (1994) Information coding in the olfactory system: evidence for a stereotyped and highly organized epitope map in the olfactory bulb. Cell 79:1245-1255

9. Mombaerts P, Wang F, Dulac C, Chao SK, Nemes A, Mendelsohn M (1996) Visualizing an olfactory sensory map. Cell $87: 675-686$

10. Malnic B, Hirono J, Sato T, Buck LB (1999) Combinatorial receptor codes for odors. Cell 96:713-723

11. Cleland T, Linster C (2003) Central olfactory processing. In: Doty R (ed) Handbook of olfaction and gustation. Dekker, New York, pp 165-181

12. Johnson BA, Leon M (2000) Modular representations of odorants in the glomerular layer of the rat olfactory bulb and the effects of stimulus concentration. J Comp Neurol 422:496-509

13. Chaigneau E, Tiret P, Lecoq J, Ducros M, Knöpfel T, Charpak S (2007) The relationship between blood flow and neuronal activity in the rodent olfactory bulb. J Neurosci 27:6452-6460

14. Bailey MS, Shipley MT (1993) Astrocyte subtypes in the rat olfactory bulb: morphological heterogeneity and differential laminar distribution. J Comp Neurol 328:501-526

15. Petzold GC, Albeanu DF, Sato TF, Murthy VN (2008) Coupling of neural activity to blood flow in olfactory glomeruli is mediated by astrocytic pathways. Neuron 58:897-910

16. Coopersmith R, Leon M (1987) Glycogen phosphorylase activity in the olfactory bulb of the young rat. J Comp Neurol 261:148-154 
17. Nawroth JC, Greer CA, Chen WR, Laughlin SB, Shepherd GM (2007) An energy budget for the olfactory glomerulus. J Neurosci 27:9790-9800

18. Ames A (2000) CNS energy metabolism as related to function. Brain Res Brain Res Rev 34:42-68

19. Sokoloff L, Reivich M, Kennedy C, Des Rosiers MH, Patlak CS, Pettigrew KD (1977) The [14C]deoxyglucose method for the measurement of local cerebral glucose utilization: theory, procedure, and normal values in the conscious and anesthetized albino rat. J Neurochem 28:897-916

20. Sharp FR, Kauer JS, Shepherd GM (1977) Laminar analysis of 2-deoxyglucose uptake in olfactory bulb and olfactory cortex of rabbit and rat. J Neurophysiol 40:800-813

21. Stewart WB, Kauer JS, Shepherd GM (1979) Functional organization of rat olfactory bulb analysed by the 2-deoxyglucose method. J Comp Neurol 185:715-734

22. Coopersmith R, Leon M (1984) Enhanced neural response to familiar olfactory cues. Science 225:849-851

23. Johnson BA, Woo CC, Hingco EE, Pham KL, Leon M (1999) Multidimensional chemotopic responses to n-aliphatic acid odorants in the rat olfactory bulb. J Comp Neurol 409:529-548

24. Johnson BA, Leon M (2000) Odorant molecular length: one aspect of the olfactory code. J Comp Neurol 426:330-338

25. Johnson BA, Farahbod H, Leon M (2005) Interactions between odorant functional group and hydrocarbon structure influence activity in glomerular response modules in the rat olfactory bulb. J Comp Neurol 483:205-216

26. Linster C, Johnson BA, Yue E, Morse A, Xu Z, Hingco EE (2001) Perceptual correlates of neural representations evoked by odorant enantiomers. J Neurosci 21:9837-9843

27. Linster C, Johnson BA, Morse A, Yue E, Leon M (2002) Spontaneous versus reinforced olfactory discriminations. J Neurosci 22:6842-6845

28. Wree A, Schleicher A (1988) The determination of the local cerebral glucose utilization with the 2-deoxyglucose method. Histochemistry 90:109-121

29. Miller S, Coopersmith R, Leon M (1991) Biochemical quantitation and histochemical localization of glucose-6-phosphate dehydrogenase activity in the olfactory system of adult and aged rats. Neurochem Res 16:475-481

30. Woo CC, Hingco EE, Johnson BA, Leon M (2007) Broad activation of the glomerular layer enhances subsequent olfactory responses. Chem Senses 32:51-55

31. Fang Q, Sakadzić S, Ruvinskaya L, Devor A, Dale AM, Boas DA (2008) Oxygen advection and diffusion in a three-dimensional vascular anatomical network. Opt Express 16:17530-17541

32. Sutton BP, Ouyang C, Karampinos DC, Miller GA (2009) Current trends and challenges in MRI acquisitions to investigate brain function. Int J Psychophysiol 73:33-42

33. Ogawa S, Lee TM, Kay AR, Tank DW (1990) Brain magnetic resonance imaging with contrast dependent on blood oxygenation. Proc Natl Acad Sci U S A 87:9868-9872

34. Logothetis NK, Pauls J, Augath M, Trinath T, Oeltermann A (2001) Neurophysiological investigation of the basis of the fMRI signal. Nature 412:150-157

35. Aubert A, Pellerin L, Magistretti PJ, Costalat R (2007) A coherent neurobiological framework for functional neuroimaging provided by a model integrating compartmentalized energy metabolism. Proc Natl Acad Sci U S A 104:4188-4193

36. Yang X, Renken R, Hyder F, Siddeek M, Greer CA, Shepherd GM (1998) Dynamic mapping at the laminar level of odorelicited responses in rat olfactory bulb by functional MRI. Proc Natl Acad Sci U S A 95:7715-7720

37. Xu F, Kida I, Hyder F, Shulman RG (2000) Assessment and discrimination of odor stimuli in rat olfactory bulb by dynamic functional MRI. Proc Natl Acad Sci U S A 97:10601-10606
38. Kida I, Xu F, Shulman RG, Hyder F (2002) Mapping at glomerular resolution: fMRI of rat olfactory bulb. Magn Reson Med 48:570-576

39. Schafer JR, Kida I, Xu F, Rothman DL, Hyder F (2006) Reproducibility of odor maps by fMRI in rodents. Neuroimage 31:1238-1246

40. Xu F, Liu N, Kida I, Rothman DL, Hyder F, Shepherd GM (2003) Odor maps of aldehydes and esters revealed by functional MRI in the glomerular layer of the mouse olfactory bulb. Proc Natl Acad Sci U S A 100:11029-11034

41. Schafer JR, Kida I, Rothman DL, Hyder F, Xu F (2005) Adaptation in the rodent olfactory bulb measured by fMRI. Magn Reson Med 54:443-448

42. Martin C, Grenier D, Thévenet M, Vigouroux M, Bertrand B, Janier M (2007) fMRI visualization of transient activations in the rat olfactory bulb using short odor stimulations. Neuroimage 36:1288-1293

43. Grinvald A, Lieke E, Frostig RD, Gilbert CD, Wiesel TN (1986) Functional architecture of cortex revealed by optical imaging of intrinsic signals. Nature 324:361-364

44. Rubin BD, Katz LC (1999) Optical imaging of odorant representations in the mammalian olfactory bulb. Neuron 23:499-511

45. Meister M, Bonhoeffer T (2001) Tuning and topography in an odor map on the rat olfactory bulb. J Neurosci 21:1351-1360

46. Uchida N, Takahashi YK, Tanifuji M, Mori K (2000) Odor maps in the mammalian olfactory bulb: domain organization and odorant structural features. Nat Neurosci 3:1035-1043

47. Belluscio L, Katz LC (2001) Symmetry, stereotypy, and topography of odorant representations in mouse olfactory bulbs. J Neurosci 21:2113-2122

48. Luo M, Katz LC (2001) Response correlation maps of neurons in the mammalian olfactory bulb. Neuron 32:1165-1179

49. Ratzlaff EH, Grinvald A (1991) A tandem-lens epifluorescence macroscope: hundred-fold brightness advantage for wide-field imaging. J Neurosci Methods 36:127-137

50. Harrison TC, Sigler A, Murphy TH (2009) Simple and cost-effective hardware and software for functional brain mapping using intrinsic optical signal imaging. J Neurosci Methods 182:211-218

51. Soucy ER, Albeanu DF, Fantana AL, Murthy VN, Meister M (2009) Precision and diversity in an odor map on the olfactory bulb. Nat Neurosci 12:210-220

52. Oka Y, Takai Y, Touhara K (2009) Nasal airflow rate affects the sensitivity and pattern of glomerular odorant responses in the mouse olfactory bulb. J Neurosci 29:12070-12078

53. Takahashi YK, Nagayama S, Mori K (2004) Detection and masking of spoiled food smells by odor maps in the olfactory bulb. J Neurosci 24:8690-8694

54. Rubin BD, Katz LC (2001) Spatial coding of enantiomers in the rat olfactory bulb. Nat Neurosci 4:355-356

55. Grossman KJ, Mallik AK, Ross J, Kay LM, Issa NP (2008) Glomerular activation patterns and the perception of odor mixtures. Eur J Neurosci 27:2676-2685

56. Lin DY, Zhang S, Block E, Katz LC (2005) Encoding social signals in the mouse main olfactory bulb. Nature 434:470-477

57. Lin DY, Shea SD, Katz LC (2006) Representation of natural stimuli in the rodent main olfactory bulb. Neuron 50:937-949

58. Frostig RD, Lieke EE, Ts'o DY, Grinvald A (1990) Cortical functional architecture and local coupling between neuronal activity and the microcirculation revealed by in vivo high-resolution optical imaging of intrinsic signals. Proc Natl Acad Sci U S A 87:6082-6086

59. MacVicar BA, Hochman D (1991) Imaging of synaptically evoked intrinsic optical signals in hippocampal slices. J Neurosci $11: 1458-1469$

60. Cohen LB, Hille B, Keynes RD (1970) Changes in axon birefringence during the action potential. J Physiol 211:495-515 
61. Cohen LB, Keynes RD, Landowne D (1972) Changes in light scattering that accompany the action potential in squid giant axons: potential-dependent components. J Physiol 224:701-725

62. Tomita M, Ohtomo M, Suzuki N (2006) Contribution of the flow effect caused by shear-dependent RBC aggregation to NIR spectroscopic signals. Neuroimage 33:1-10

63. Aitken PG, Fayuk D, Somjen GG, Turner DA (1999) Use of intrinsic optical signals to monitor physiological changes in brain tissue slices. Methods 18:91-103

64. Fayuk D, Aitken PG, Somjen GG, Turner DA (2002) Two different mechanisms underlie reversible, intrinsic optical signals in rat hippocampal slices. J Neurophysiol 87:1924-1937

65. Gurden H, Uchida N, Mainen ZF (2006) Sensory-evoked intrinsic optical signals in the olfactory bulb are coupled to glutamate release and uptake. Neuron 52:335-345

66. Kadekaro M, Crane AM, Sokoloff L (1985) Differential effects of electrical stimulation of sciatic nerve on metabolic activity in spinal cord and dorsal root ganglion in the rat. Proc Natl Acad Sci U S A 82:6010-6013

67. Thomsen K, Offenhauser N, Lauritzen M (2004) Principal neuron spiking: neither necessary nor sufficient for cerebral blood flow in rat cerebellum. J Physiol 560:181-189

68. Uchida N, Mainen ZF (2003) Speed and accuracy of olfactory discrimination in the rat. Nat Neurosci 6:1224-1229

69. Abraham NM, Spors H, Carleton A, Margrie TW, Kuner T, Schaefer AT (2004) Maintaining accuracy at the expense of speed: stimulus similarity defines odor discrimination time in mice. Neuron 44:865-876

70. Gupta RK, Salzberg BM, Grinvald A, Cohen LB, Kamino K, Lesher S (1981) Improvements in optical methods for measuring rapid changes in membrane potential. J Membr Biol 58:123-137

71. Shoham D, Glaser DE, Arieli A, Kenet T, Wijnbergen C, Toledo Y (1999) Imaging cortical dynamics at high spatial and temporal resolution with novel blue voltage-sensitive dyes. Neuron 24:791-802

72. Spors H, Grinvald A (2002) Spatio-temporal dynamics of odor representations in the mammalian olfactory bulb. Neuron 34:301-315

73. Orbach HS, Cohen LB (1983) Optical monitoring of activity from many areas of the in vitro and in vivo salamander olfactory bulb: a new method for studying functional organization in the vertebrate central nervous system. J Neurosci 3:2251-2262

74. Buonviso N, Amat C, Litaudon P, Roux S, Royet J, Farget V (2003) Rhythm sequence through the olfactory bulb layers during the time window of a respiratory cycle. Eur $\mathbf{J}$ Neurosci 17:1811-1819

75. Neville KR, Haberly LB (2003) Beta and gamma oscillations in the olfactory system of the urethane-anesthetized rat. J Neurophysiol 90:3921-3930

76. Friedrich RW, Korsching SI (1997) Combinatorial and chemotopic odorant coding in the zebrafish olfactory bulb visualized by optical imaging. Neuron 18:737-752

77. Wachowiak M, Cohen LB (2001) Representation of odorants by receptor neuron input to the mouse olfactory bulb. Neuron 32:723-735

78. Fried HU, Fuss SH, Korsching SI (2002) Selective imaging of presynaptic activity in the mouse olfactory bulb shows concentration and structure dependence of odor responses in identified glomeruli. Proc Natl Acad Sci U S A 99:3222-3227

79. Verhagen JV, Wesson DW, Netoff TI, White JA, Wachowiak M (2007) Sniffing controls an adaptive filter of sensory input to the olfactory bulb. Nat Neurosci 10:631-639

80. Wachowiak M, Cohen LB (2003) Correspondence between odorant-evoked patterns of receptor neuron input and intrinsic optical signals in the mouse olfactory bulb. J Neurophysiol 89:1623-1639
81. Spors H, Wachowiak M, Cohen LB, Friedrich RW (2006) Temporal dynamics and latency patterns of receptor neuron input to the olfactory bulb. J Neurosci 26:1247-1259

82. McGann JP, Pírez N, Gainey MA, Muratore C, Elias AS, Wachowiak M (2005) Odorant representations are modulated by intra- but not interglomerular presynaptic inhibition of olfactory sensory neurons. Neuron 48:1039-1053

83. Kepecs A, Uchida N, Mainen ZF (2006) The sniff as a unit of olfactory processing. Chem Senses 31:167-179

84. Wesson DW, Carey RM, Verhagen JV, Wachowiak M (2008) Rapid encoding and perception of novel odors in the rat. PLoS Biol 6:e82

85. Carey RM, Verhagen JV, Wesson DW, Pírez N, Wachowiak M (2009) Temporal structure of receptor neuron input to the olfactory bulb imaged in behaving rats. J Neurophysiol 101:1073-1088

86. Wesson DW, Verhagen JV, Wachowiak M (2009) Why sniff fast? The relationship between sniff frequency, odor discrimination, and receptor neuron activation in the rat. J Neurophysiol 101:1089-1102

87. Bozza T, McGann JP, Mombaerts P, Wachowiak M (2004) In vivo imaging of neuronal activity by targeted expression of a genetically encoded probe in the mouse. Neuron 42:9-21

88. Wachowiak M, McGann JP, Heyward PM, Shao Z, Puche AC, Shipley MT (2005) Inhibition [corrected] of olfactory receptor neuron input to olfactory bulb glomeruli mediated by suppression of presynaptic calcium influx. J Neurophysiol 94:2700 2712

89. Bathellier B, Van De Ville D, Blu T, Unser M, Carleton A (2007) Wavelet-based multi-resolution statistics for optical imaging signals: application to automated detection of odour activated glomeruli in the mouse olfactory bulb. Neuroimage 34:1020-1035

90. Yaksi E, Friedrich RW (2006) Reconstruction of firing rate changes across neuronal populations by temporally deconvolved Ca2+ imaging. Nat Methods 5:377-383

91. Aroniadou-Anderjaska V, Zhou FM, Priest CA, Ennis M, Shipley MT (2000) Tonic and synaptically evoked presynaptic inhibition of sensory input to the rat olfactory bulb via GABA(B) heteroreceptors. J Neurophysiol 84:1194-1203

92. Vucinić D, Cohen LB, Kosmidis EK (2006) Interglomerular center-surround inhibition shapes odorant-evoked input to the mouse olfactory bulb in vivo. J Neurophysiol 95:1881-1887

93. Pírez N, Wachowiak M (2008) In vivo modulation of sensory input to the olfactory bulb by tonic and activity-dependent presynaptic inhibition of receptor neurons. J Neurosci 28:6360-6371

94. Petzold GC, Hagiwara A, Murthy VN (2009) Serotonergic modulation of odor input to the mammalian olfactory bulb. Nat Neurosci 12:784-791

95. Aungst JL, Heyward PM, Puche AC, Karnup SV, Hayar A, Szabo G (2003) Centre-surround inhibition among olfactory bulb glomeruli. Nature 426:623-629

96. Nakai J, Ohkura M, Imoto K (2001) A high signal-to-noise $\mathrm{Ca} 2+$ probe composed of a single green fluorescent protein. Nat Biotechnol 19:137-141

97. Díez-García J, Matsushita S, Mutoh H, Nakai J, Ohkura M, Yokoyama J (2005) Activation of cerebellar parallel fibers monitored in transgenic mice expressing a fluorescent $\mathrm{Ca} 2+$ indicator protein. Eur J Neurosci 22:627-635

98. Fletcher ML, Masurkar AV, Xing J, Imamura F, Xiong W, Nagayama S (2009) Optical imaging of postsynaptic odor representation in the glomerular layer of the mouse olfactory bulb. J Neurophysiol 102:817-830

99. De Saint Jan D, Hirnet D, Westbrook GL, Charpak S (2009) External tufted cells drive the output of olfactory bulb glomeruli. J Neurosci 29:2043-2052 
100. Silva AC, Lee JH, Aoki I, Koretsky AP (2004) Manganeseenhanced magnetic resonance imaging (MEMRI): methodological and practical considerations. NMR Biomed 17:532-543

101. Itoh K, Sakata M, Watanabe M, Aikawa Y, Fujii H (2008) The entry of manganese ions into the brain is accelerated by the activation of $\mathrm{N}$-methyl-d-aspartate receptors. Neuroscience 154:732-740

102. Pautler RG, Koretsky AP (2002) Tracing odor-induced activation in the olfactory bulbs of mice using manganese-enhanced magnetic resonance imaging. Neuroimage 16:441-448

103. Eschenko O, Canals S, Simanova I, Logothetis NK (2010) Behavioral, electrophysiological and histopathological consequences of systemic manganese administration in MEMRI. Magn Reson Imaging 28:1165-1174

104. Chuang K, Belluscio L, Koretsky AP (2010) In vivo detection of individual glomeruli in the rodent olfactory bulb using manganese enhanced MRI. Neuroimage 49:1350-1356

105. Helmchen F, Denk W (2005) Deep tissue two-photon microscopy. Nat Methods 2:932-940

106. Wachowiak M, Denk W, Friedrich RW (2004) Functional organization of sensory input to the olfactory bulb glomerulus analyzed by two-photon calcium imaging. Proc Natl Acad Sci U S A 101:9097-9102

107. Lecoq J, Tiret P, Najac M, Shepherd GM, Greer CA, Charpak S (2009) Odor-evoked oxygen consumption by action potential and synaptic transmission in the olfactory bulb. J Neurosci 29:1424-1433

108. Lebedev AY, Cheprakov AV, Sakadzić S, Boas DA, Wilson DF, Vinogradov SA (2009) Dendritic phosphorescent probes for oxygen imaging in biological systems. ACS Appl Mater Interfaces 1:1292-1304

109. Sakadzić S, Roussakis E, Yaseen MA, Mandeville ET, Srinivasan VJ, Arai K (2010) Two-photon high-resolution measurement of partial pressure of oxygen in cerebral vasculature and tissue. Nat Methods 7:755-759

110. Kleinfeld D, Mitra PP, Helmchen F, Denk W (1998) Fluctuations and stimulus-induced changes in blood flow observed in individual capillaries in layers 2 through 4 of rat neocortex. Proc Natl Acad Sci U S A 95:15741-15746

111. Tiret P, Chaigneau E, Lecoq J, Charpak S (2009) Two-photon imaging of capillary blood flow in olfactory bulb glomeruli. Methods Mol Biol 489:81-91

112. Chaigneau E, Oheim M, Audinat E, Charpak S (2003) Twophoton imaging of capillary blood flow in olfactory bulb glomeruli. Proc Natl Acad Sci U S A 100:13081-13086

113. Lecoq J, Tiret $P$, Charpak S (2009) Peripheral adaptation codes for high odor concentration in glomeruli. J Neurosci 29:3067-3072

114. Lauritzen M, Fabricius M (1995) Real time laser-Doppler perfusion imaging of cortical spreading depression in rat neocortex. Neuroreport 6:1271-1273

115. Ances BM, Greenberg JH, Detre JA (1999) Laser doppler imaging of activation-flow coupling in the rat somatosensory cortex. Neuroimage 10:716-723

116. Dunn AK, Bolay H, Moskowitz MA, Boas DA (2001) Dynamic imaging of cerebral blood flow using laser speckle. J Cereb Blood Flow Metab 21:195-201

117. Boas DA, Dunn AK (2010) Laser speckle contrast imaging in biomedical optics. J Biomed Opt 15:011109

118. Dunn AK, Devor A, Bolay H, Andermann ML, Moskowitz MA, Dale AM (2003) Simultaneous imaging of total cerebral hemoglobin concentration, oxygenation, and blood flow during functional activation. Opt Lett 28:28-30

119. Durduran T, Burnett MG, Yu G, Zhou C, Furuya D, Yodh AG (2004) Spatiotemporal quantification of cerebral blood flow during functional activation in rat somatosensory cortex using laser-speckle flowmetry. J Cereb Blood Flow Metab 24:518-525

120. Devor A, Ulbert I, Dunn AK, Narayanan SN, Jones SR, Andermann ML (2005) Coupling of the cortical hemodynamic response to cortical and thalamic neuronal activity. Proc Natl Acad Sci U S A 102:3822-3827

121. Devor A, Hillman EMC, Tian P, Waeber C, Teng IC, Ruvinskaya L (2008) Stimulus-induced changes in blood flow and 2-deoxyglucose uptake dissociate in ipsilateral somatosensory cortex. J Neurosci 28:14347-14357

122. Jones PB, Shin HK, Boas DA, Hyman BT, Moskowitz MA, Ayata C (2008) Simultaneous multispectral reflectance imaging and laser speckle flowmetry of cerebral blood flow and oxygen metabolism in focal cerebral ischemia. J Biomed Opt 13:044007

123. Li P, Ni S, Zhang L, Zeng S, Luo Q (2006) Imaging cerebral blood flow through the intact rat skull with temporal laser speckle imaging. Opt Lett 31:1824-1826

124. Duncan DD, Kirkpatrick SJ (2008) Can laser speckle flowmetry be made a quantitative tool? J Opt Soc Am A Opt Image Sci Vis 25:2088-2094

125. Maheswari RU, Takaoka H, Kadono H, Homma R, Tanifuji M (2003) Novel functional imaging technique from brain surface with optical coherence tomography enabling visualization of depth resolved functional structure in vivo. J Neurosci Methods 124:83-92

126. Chen Y, Aguirre AD, Ruvinskaya L, Devor A, Boas DA, Fujimoto JG (2009) Optical coherence tomography (OCT) reveals depth-resolved dynamics during functional brain activation. J Neurosci Methods 178:162-173

127. Srinivasan VJ, Sakadzić S, Gorczynska I, Ruvinskaya S, Wu W, Fujimoto JG (2009) Depth-resolved microscopy of cortical hemodynamics with optical coherence tomography. Opt Lett 34:3086-3088

128. Srinivasan VJ, Sakadzić S, Gorczynska I, Ruvinskaya S, Wu W, Fujimoto JG (2010) Quantitative cerebral blood flow with optical coherence tomography. Opt Express 18:2477-2494

129. Srinivasan VJ, Jiang JY, Yaseen MA, Radhakrishnan H, Wu W, Barry S (2010) Rapid volumetric angiography of cortical microvasculature with optical coherence tomography. Opt Lett $35: 43-45$

130. Wang RK, An L (2009) Doppler optical micro-angiography for volumetric imaging of vascular perfusion in vivo. Opt Express 17:8926-8940

131. Fletcher ML, Chen WR (2010) Neural correlates of olfactory learning: critical role of centrifugal neuromodulation. Learn Mem 17:561-570

132. Fantana AL, Soucy ER, Meister M (2008) Rat olfactory bulb mitral cells receive sparse glomerular inputs. Neuron 59:802-814

133. Friedrich RW, Laurent G (2001) Dynamic optimization of odor representations by slow temporal patterning of mitral cell activity. Science 291:889-894

134. Wachowiak M, Shipley MT (2006) Coding and synaptic processing of sensory information in the glomerular layer of the olfactory bulb. Semin Cell Dev Biol 17:411-423 